\title{
Ion-induced nucleation. II. Polarizable multipolar molecules
}

\author{
I. Kusaka, Z.-G. Wang, and J. H. Seinfeld \\ Department of Chemical Engineering, California Institute of Technology, Pasadena, California 91125
}

(Received 11 July 1995; accepted 18 August 1995)

\begin{abstract}
Density functional theory is applied to ion-induced nucleation of polarizable multipolar molecules. The asymmetric nature of the ion-molecule interaction is shown to cause the sign preference in ion-induced nucleation. When the ion-molecule interaction is weak, the observed sign preference is consistent with that of the bare ion-molecule interaction potential and decreases with increasing supersaturation. However, as the ion-molecule interaction becomes stronger, the sign preference in the reversible work exhibits some nontrivial behavior. For molecular parameters applicable for $\mathrm{CS}_{2}$ and $\mathrm{CH}_{4}$, the predicted values of the reversible work of nucleation depend on the sign of the ion charge, yielding a difference in the nucleation rate by factors of 10 to $10^{2}$ and 10 to $10^{5}$, respectively. (C) 1995 American Institute of Physics.
\end{abstract}

\section{INTRODUCTION}

In ion-induced nucleation ions act as sites for vapor molecule cluster formation, thereby enhancing the ease with which clusters can form in a supersaturated vapor over that in the absence of ions. ${ }^{1-7}$ Despite the long recognition of the phenomenon of ion-induced nucleation, a detailed understanding of the physics of the interaction between neutral vapor molecules and ions that leads to an enhanced rate of nucleation in the presence of ions has been lacking. A question basic to the process is whether one can predict on fundamental grounds how the rate of nucleation will, for a particular vapor molecule, depend on the sign and properties of the ion. While both positive and negative ions increase the nucleation rate, most substances exhibit a preference for one ion polarity over the other. For some substances, however, even a qualitative agreement among experiments on the sign preference for nucleation is lacking. ${ }^{8}$ Definite conclusion on the sign preference for some substances still awaits further investigations. ${ }^{9}$

Physically, the dependence of the ion-induced nucleation rate of a substance on the sign of the ion charge must arise from some sort of asymmetry in the molecular interactions. Such asymmetry should, in principle, manifest itself in a sign dependence of the relevant thermodynamic quantities such as the surface tension. Several attempts have been made to incorporate molecular characteristics within the framework of the capillarity theory. ${ }^{4,10-13}$ These theories were critically reviewed by Rabeony and Mirabel, ${ }^{8}$ who concluded that only Rusanov and Kuni's model ${ }^{12,13}$ could correctly predict the sign preference for a few substances, although the predicted sign effect was shown to be extremely sensitive to a parameter in the theory that cannot be evaluated within the classical framework. It has also been noted ${ }^{8}$ that, except for its inability to explain the sign effect, the best predictions of the reversible work, when compared with experimental data, come from Thomson's original equation. ${ }^{14}$ Most importantly, these theories all apply for polar materials and are incapable of predicting a sign preference for nonpolar substances. The failure of these approaches merely points to the need for consistent treatment of the molecular characteristics in evaluating the free energy by means of statistical mechanics.

In the previous work, ${ }^{15}$ we applied a statistical mechani- cal density functional theory to ion-induced nucleation of dipolar molecules. Asymmetry was introduced into the ionmolecule interaction by means of a permanent dipole moment placed at a distance $a$ off the center of a molecule. It was concluded that this asymmetry in the ion-molecule interaction is directly responsible for the sign preference in ion-induced nucleation.

This paper is intended to propose an alternative mechanism, applicable for both polar and nonpolar substances, through which a sign preference in the rate of nucleation arises. In particular, we present a density functional theory for ion-induced nucleation of polarizable multipolar molecules. For a fixed orientation of a molecule, the ionmolecule interaction through the molecular polarizability is independent of the sign of the ion charge, while that through the permanent multipole moments is not. As a result of this asymmetry, the reversible work acquires a dependence on the sign of the ion charge.

The outline of this paper is as follows. In Sec. II, we first introduce our model representation of a molecule, and then construct a density functional for the grand potential in terms of two order parameters, the particle number density and the rescaled ion charge. The latter is related in a simple way to a locally defined dielectric constant. Bulk properties are derived from the density functional. Section III describes the solution methods for determining the equilibrium profiles. The reversible work of nucleation is obtained from the equilibrium profiles and reported in Sec. IV. Finally, some concluding remarks are given with a brief discussion in Sec. V.

\section{DENSITY FUNCTIONAL THEORY}

\section{A. The model and the intermolecular potential}

Let us consider a system of spherical molecules each of which has, at its center, electric permanent multipole moments, polarizability, and hyperpolarizabilities. We assume that a molecule is in its ground state under the influence of the external electric field and suppose that the interaction potential $\phi(1,2)$ between one molecule at $\mathbf{r}_{1}$ with orientation $\hat{R}_{1}$ and the other at $\mathbf{r}_{2}$ with orientation $\hat{R}_{2}$ can be written as

$$
\phi(1,2)=\phi^{d}\left(r_{12}\right)+\phi^{\text {att }}\left(r_{12}\right)+\phi^{\mathrm{mp}}(1,2),
$$


where $r_{12}=\left|\mathbf{r}_{2}-\mathbf{r}_{1}\right|$. We use the notation $j$ to represent both the translational and orientational coordinates of molecule $j$. $\phi^{d}\left(r_{12}\right)$ and $\phi^{\text {att }}\left(r_{12}\right)$ form the isotropic part of the interaction potential $\phi(1,2) . \phi^{d}\left(r_{12}\right)$ is the hard sphere potential given by

$$
\phi^{d}\left(r_{12}\right)=\left\{\begin{array}{cc}
+\infty & \text { if } r_{12}<d \\
0 & \text { otherwise. }
\end{array}\right.
$$

$\phi^{\text {att }}\left(r_{12}\right)$ is the perturbative attractive potential whose explicit form is chosen to be

$$
\phi^{\mathrm{att}}\left(r_{12}\right)=-\epsilon^{\mathrm{att}}\left(\frac{d}{r_{12}}\right)^{6},
$$

where $\epsilon^{\text {att }}$ is a positive constant.

In this model, the anisotropic part of $\phi(1,2)$ arises from the orientational dependence of the interaction potential between multipole moments on the molecule 1 and those on the molecule 2. Care must be taken, however, to correctly incorporate the effect of the molecular polarizability. To determine the explicit form of this anisotropic interaction potential $\phi^{\mathrm{mp}}(1,2)$, we first consider the electrostatic energy $U(1)$, in the external electric field $F_{\alpha}(\mathbf{r})$, of a neutral molecule 1 in a fixed position and orientation. In this external field, the molecule acquires the induced multipole moments, which along with its permanent multipole moments constitute the total multipole moments. Thus ${ }^{16,17}$

$$
\begin{aligned}
U(1)= & U^{\mathrm{pol}}(1)-\mu_{\alpha}^{(T)}(1) F_{\alpha}\left(\mathbf{r}_{1}\right)-\frac{1}{3} \Theta_{\alpha \beta}^{(T)}(1) F_{\alpha \beta}\left(\mathbf{r}_{1}\right) \\
& -\frac{1}{15} \Omega_{\alpha \beta \gamma}^{(T)}(1) F_{\alpha \beta \gamma}\left(\mathbf{r}_{1}\right)-\frac{1}{105} \Phi_{\alpha \beta \gamma \delta}^{(T)}(1) F_{\alpha \beta \gamma \delta}\left(\mathbf{r}_{1}\right) \\
& + \text { h.o., }
\end{aligned}
$$

where tensor notation is employed and

$$
\begin{aligned}
U^{\mathrm{pol}}(1) \equiv & \frac{1}{2} \alpha_{\alpha \beta}(1) F_{\alpha}\left(\mathbf{r}_{1}\right) F_{\beta}\left(\mathbf{r}_{1}\right) \\
& +\frac{1}{3} A_{\alpha, \beta \gamma}(1) F_{\alpha}\left(\mathbf{r}_{1}\right) F_{\beta \gamma}\left(\mathbf{r}_{1}\right)
\end{aligned}
$$

is the work required to polarize the molecule. $\alpha_{\alpha \beta}$ and $A_{\gamma, \alpha \beta}$ are, respectively, polarizability and hyperpolarizability and are symmetric in $\alpha$ and $\beta$. Also, $A_{\gamma, \alpha \alpha \equiv 0}{ }^{16,17}$ Other terms in Eq. (4) are the electrostatic interaction energy between the resulting total multipole moments on the molecule 1 and the external electric field. $\mu_{\alpha}^{(T)}(1), \Theta_{\alpha \beta}^{(T)}(1), \Omega_{\alpha \beta \gamma}^{(T)}(1)$, and $\Phi_{\alpha \beta \gamma \delta}^{(T)}(1)$ are, respectively, the total electric dipole, quadrupole, octopole, and hexadecapole moments, of the molecule 1 expressed in a laboratory coordinate system $O-x_{1} x_{2} x_{3}$. (See Fig. 1.) In Eqs. (4) and (5),

$$
\begin{aligned}
& \left.F_{\alpha \beta}\left(\mathbf{r}_{1}\right) \equiv \frac{\partial F_{\alpha}}{\partial x_{\beta}}\right|_{\mathbf{r}=\mathbf{r}_{1}}, \\
& \left.F_{\alpha \beta \gamma}\left(\mathbf{r}_{1}\right) \equiv \frac{\partial^{2} F_{\alpha}}{\partial x_{\beta} \partial x_{\gamma}}\right|_{\mathbf{r}=\mathbf{r}_{1}}, \\
& \left.F_{\alpha \beta \gamma \delta}\left(\mathbf{r}_{1}\right) \equiv \frac{\partial^{3} F_{\alpha}}{\partial x_{\beta} \partial x_{\gamma} \partial x_{\delta}}\right|_{\mathbf{r}=\mathbf{r}_{1}}
\end{aligned}
$$

are the spatial gradients of $F_{\alpha}(\mathbf{r})$ evaluated at the center of the molecule 1. $x_{\alpha}$ is the $\alpha$ component of $\mathbf{r}$. When the electric field $F_{\alpha}(\mathbf{r})$ is due only to a point charge, h.o. in Eq. (4)

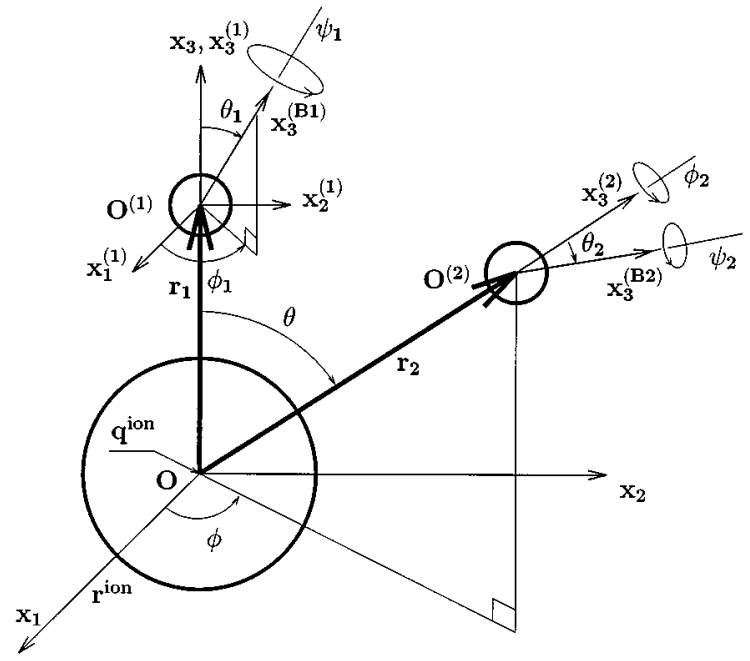

FIG. 1. Model of a molecule and an ion. The origin of the laboratory coordinate system $O-x_{1} x_{2} x_{3}$ is taken at the center of the ion, where a point charge $q^{\text {ion }}$ is located. $O^{(1)}-x_{1}^{(1)} x_{2}^{(1)} x_{3}^{(1)}$ is the local coordinate system whose origin coincides with the center of the molecule 1 , and the $x_{3}^{(1)}$ axis is parallel to $x_{3}$ the axis. $\left(\phi_{1}, \theta_{1}, \psi_{1}\right)$ is the Euler angle of the body fixed coordinate system $O^{(B 1)}-x_{1}^{(B 1)} x_{2}^{(B 1)} x_{3}^{(B 1)}$.

represents the terms $O\left(R^{-6}\right)$ or smaller, where $R$ temporarily denotes the distance between the point charge and a molecule. In Appendix A, the total multipole moments are related to the polarizabilities and the permanent multipole moments.

In our study of ion-induced nucleation, we may decompose $F_{\alpha}$ into $F_{\alpha}^{\text {ion }}$ due to an ion and $F_{\alpha}^{(j)}$ due to total multipole moments on the molecule $j$. Thus

$$
U(1)=U^{\mathrm{pol}}(1)+U^{\mathrm{ion}}(1)+\sum_{j \neq 1} U^{\mathrm{mp}}(1, j),
$$

where

$$
\begin{aligned}
U^{\text {ion }}(1)= & -\mu_{\alpha}^{(T)} F_{\alpha}^{\text {ion }}\left(\mathbf{r}_{1}\right)-\frac{1}{3} \Theta_{\alpha \beta}^{(T)}(1) F_{\alpha \beta}^{\text {ion }}\left(\mathbf{r}_{1}\right)-\frac{1}{15} \Omega_{\alpha \beta \gamma}^{(T)}(1) \\
& \times F_{\alpha \beta \gamma}^{\text {ion }}\left(\mathbf{r}_{1}\right)-\frac{1}{105} \Phi_{\alpha \beta \gamma \delta}^{(T)}(1) F_{\alpha \beta \gamma \delta}^{\text {ion }}\left(\mathbf{r}_{1}\right)+\text { h.o. }
\end{aligned}
$$

and

$$
\begin{aligned}
U^{\mathrm{mp}}(1,2)= & -\mu_{\alpha}^{(T)}(1) F_{\alpha}^{(2)}\left(\mathbf{r}_{1}\right)-\frac{1}{3} \Theta_{\alpha \beta}^{(T)}(1) F_{\alpha \beta}^{(2)}\left(\mathbf{r}_{1}\right) \\
& -\frac{1}{15} \Omega_{\alpha \beta \gamma}^{(T)}(1) F_{\alpha \beta \gamma}^{(2)}\left(\mathbf{r}_{1}\right)-\frac{1}{105} \Phi_{\alpha \beta \gamma \delta}^{(T)}(1) \\
& \times F_{\alpha \beta \gamma \delta}^{(2)}\left(\mathbf{r}_{1}\right)+\text { h.o. }
\end{aligned}
$$

Then, $U^{\mathrm{pol}}(1)+U^{\mathrm{ion}}(1)$ can be regarded as an external potential of the molecule 1 and $U^{\mathrm{mp}}(1,2)$ can be identified with $\phi^{\mathrm{mp}}(1,2)$. The explicit expression for $U^{\mathrm{mp}}(1,2)$ is given in Appendix B with its derivation. In what follows, it is enough to note that $U^{\mathrm{mp}}(1,2)$ is a sum of terms proportional to a product of a total multipole moment of the molecule 1 and that of the molecule 2 and is symmetric in 1 and 2 .

It should be noted here that the separation implied by Eq. (7) is only formal at this stage. In fact, neither $U^{\mathrm{pol}}(1)$ nor $U^{\text {ion }}(1)$ is a one body potential since $F_{\alpha}\left(\mathbf{r}_{1}\right)$ and the total multipole moments at the molecule 1 depend on the electric field due to other molecules as well as on $F_{\alpha}^{\mathrm{ion}}\left(\mathbf{r}_{1}\right)$. 


\section{B. Construction of the functional}

Consider an open system, for which the grand potential $\Omega$ is the proper thermodynamic potential. In density functional theory, $\Omega$ of the system is given as a functional of order parameters such as the position-orientation distribution function $\rho(\mathbf{r}, \hat{R})$ defined by

$$
\rho(\mathbf{r}, \hat{R}) \equiv\left\langle\sum_{j=1}^{N} \delta\left(\mathbf{r}-\mathbf{r}_{j}\right) \delta\left(\hat{R}-\hat{R}_{j}\right)\right\rangle .
$$

Within the framework of mean field theory and under the local density approximation, $\Omega$ is given in terms of $\rho(\mathbf{r}, \hat{R})$ by ${ }^{15,18}$

$$
\begin{aligned}
\Omega[\rho]= & k_{B} T \int d 1 \rho(1)\left\{\log \left[\Lambda^{3} \rho(1)\right]-1\right\} \\
& +\int d \mathbf{r}_{1} f^{\operatorname{exc}}\left(n\left(\mathbf{r}_{1}\right)\right)-\int d 1 \rho(1)[\mu-v(1)] \\
& +\frac{1}{2} \iint d 1 d 2 \rho(1) \rho(2) H\left(r_{12}-d\right) \\
& \times\left[\phi^{\text {att }}\left(r_{12}\right)+\phi^{\mathrm{mp}}(1,2)\right],
\end{aligned}
$$

where $k_{B}$ is the Boltzmann constant, $T$ is temperature, $\Lambda$ is de Broglie's thermal wavelength, and $\mu$ is the chemical potential of the system. $f^{\text {exc }}(n(\mathbf{r}))$ is the excess Helmholtz free energy per unit volume, arising from hard sphere exclusion, over the free energy density of an ideal gas. Employing the Carnahan-Starling equation, ${ }^{19}$

$$
\begin{aligned}
& f^{\mathrm{exc}}(n)=k_{B} \operatorname{Tn} \frac{y(4-3 y)}{(1-y)^{2}}, \\
& y \equiv(\pi / 6) d^{3} n .
\end{aligned}
$$

$H$ is the Heaviside step function approximating the hard core exclusion between a pair of molecules.

In Eq. (11), $v(1)$ is an external field. We take an ion as a point charge $q^{\text {ion }}$ placed at the center of a hard sphere of radius $r^{\text {ion }}$, which itself is fixed at the center of a molecular cluster. Then the ion-molecule interaction can be treated as an external potential $v(1)$, which is composed of the hard core repulsive potential and $U^{\mathrm{pol}}(1)+U^{\mathrm{ion}}(1)$. As shown in Fig. 1, we take the origin of the coordinate system $O-x_{1} x_{2} x_{3}$ at the center of the ion, and choose the $x_{3}$ axis parallel to $\mathbf{r}_{1}$. Then

$$
F_{\alpha}^{\mathrm{ion}}(\mathbf{r})=q^{\mathrm{ion}} \frac{x_{\alpha}}{r^{3}},
$$

which is substituted into Eq. (8) to obtain

$$
\begin{aligned}
U^{\mathrm{ion}}(1)= & q^{\mathrm{ion}}\left\{-\frac{1}{r_{1}^{2}} \mu_{3}^{(T)}(1)+\frac{1}{r_{1}^{3}} \Theta_{33}^{(T)}(1)\right. \\
& \left.-\frac{1}{r_{1}^{4}} \Omega_{333}^{(T)}(1)+\frac{1}{r_{1}^{5}} \Phi_{3333}^{(T)}(1)\right\} \\
\equiv & q^{\mathrm{ion}} u^{T}(1) .
\end{aligned}
$$

We leave the detailed derivation of Eq. (14) to Appendix C.

\section{Approximate formulas}

The equilibrium distribution for $\rho(\mathbf{r}, \hat{R})$ is determined by the stationarity condition of the grand potential $\Omega$ :

$$
\frac{\delta \Omega}{\delta \rho}=0 .
$$

Because of the implicit dependence of $U^{\mathrm{pol}}(1)$ and $U^{\text {ion }}(1)$ on $\rho(\mathbf{r}, \hat{R})$, the functional derivative in Eq. (15) cannot be readily performed. To circumvent this difficulty, we shall introduce further approximations.

In this work, a molecule is represented as a polarizable hard sphere of radius $d / 2$ with attractive potential and permanent multipole moments grafted on it. Consider the electric field $F_{\alpha}(\mathbf{r})$ inside the molecule 1, fixed at $\mathbf{r}_{1}$, as a result of the ion and other molecules, which constitute charge distribution exterior to this hard sphere of radius $d / 2$. Note that $F_{\alpha}(\mathbf{r})$ depends parametrically on $r_{1}$ which determines the boundary of the charge distribution. Because of the axial symmetry of the system around $\mathbf{r}_{1}, F_{\alpha}(\mathbf{r})$ is given in terms of some function $f_{1}$ as

$$
F_{\alpha}\left(\mathbf{r} ; r_{1}\right)=f_{1}\left(\theta ; r_{1}\right) \frac{x_{\alpha}}{r^{3}},
$$

where $\theta$ is the angle between $\mathbf{r}$ and $\mathbf{r}_{1}$. In arriving at Eq. (16), we made use of the fact that $F_{\alpha}(\mathbf{r})$ is divergenceless inside the hard sphere. As an approximation, we ignore the $\theta$ dependence of $f_{1}$ and denote it by $q^{\text {eff }}\left(r_{1}\right)$. Then,

$$
F_{\alpha}\left(\mathbf{r} ; r_{1}\right)=q^{\mathrm{eff}}\left(r_{1}\right) \frac{x_{\alpha}}{r^{3}},
$$

which is the electric field we would have in the absence of any molecule as a result of a point charge $q^{\text {eff }}\left(r_{1}\right)$ at the origin. In other words, under the approximation we have introduced, the effect of the intermolecular interaction represented by $\phi^{\mathrm{mp}}(1,2)$ is to simply rescale the ion charge $q^{\text {ion }}$ to $q^{\text {eff }}\left(r_{1}\right)$. Equation (17) gives the electric field produced by the ion and other molecules inside the hard sphere representing the molecule 1 , in contrast to the electric displacement given by Eq. (13). Their ratio can be interpreted as a locally defined dielectric constant $\epsilon\left(r_{1}\right)$ :

$$
\epsilon\left(r_{1}\right)=\frac{q^{\text {ion }}}{q^{\text {eff }}\left(r_{1}\right)} .
$$

Using the explicit form of $F_{\alpha}(\mathbf{r})$ given by Eq. (17), we may rewrite Eqs. (4) and (5) as

$$
U(1)=U^{\mathrm{pol}}(1)+q^{\mathrm{eff}}\left(r_{1}\right) u^{T}(1)
$$

and

$$
\begin{aligned}
U^{\mathrm{pol}}(1) & =\frac{1}{2} q^{\mathrm{eff}}\left(r_{1}\right)^{2}\left(\frac{1}{r_{1}^{4}} \alpha_{33}(1)-\frac{2}{r_{1}^{5}} A_{3,33}(1)\right) \\
& \equiv \frac{1}{2} q^{\mathrm{eff}}\left(r_{1}\right)^{2} u^{\mathrm{pol}}(1),
\end{aligned}
$$

respectively. It should be noted here that the field gradients in Eqs. (4) and (5) are evaluated at the center of the molecule 1 for the fixed position of that molecule. Operationally, we 
take the spatial derivatives of the field $F_{\alpha}\left(\mathbf{r} ; r_{1}\right)$ with respect to $\mathbf{r}$ for fixed $\mathbf{r}_{1}$ and evaluate them at $\mathbf{r}_{1}$. As mentioned in Sec. II A, the total multipole moments can be expressed in terms of the permanent multipole moments and the polarizabilities; thus we may rewrite $u^{T}(1)$, defined in Eq. (14) as

$$
\begin{aligned}
u^{T}(1)= & -q^{\mathrm{eff}}\left(r_{1}\right) u^{\mathrm{pol}}(1)-\frac{1}{r_{1}^{2}} \mu_{3}^{(P)}(1)+\frac{1}{r_{1}^{3}} \Theta_{33}^{(P)}(1) \\
& -\frac{1}{r_{1}^{4}} \Omega_{333}^{(P)}(1)+\frac{1}{r_{1}^{5}} \Phi_{3333}^{(P)}(1) \\
\equiv & -q^{\mathrm{eff}}\left(r_{1}\right) u^{\mathrm{pol}}(1)+u^{P}(1) .
\end{aligned}
$$

The detailed derivation of Eqs. (19)-(21) is given in Appendix $\mathrm{C}$. We use the superscript $(P)$ for the tensor components of the permanent multipole moments. Inspection of Eqs. (14), (20), and (21) reveals that $U^{\mathrm{pol}}(1)$ and $U^{\text {ion }}(1)$ now depend only on $r_{1}$ and the orientation of the molecule 1 . Thus, we have reduced the many body potential $U^{\mathrm{pol}}(1)$ and $U^{\text {ion }}(1)$ to the corresponding effective one body potentials.

$q^{\text {eff }}(r)$ introduced above has yet to be determined. It is therefore natural to rewrite the functional for $\Omega$ so that $q^{\text {eff }}(r)$ serves as an order parameter. We first assume that $\rho(\mathbf{r}, \hat{R})$ is separable:

$$
\rho(\mathbf{r}, \hat{R})=n(\mathbf{r}) m(\mathbf{r}, \hat{R}),
$$

where

$$
n(\mathbf{r}) \equiv\left\langle\sum_{j=1}^{N} \delta\left(\mathbf{r}-\mathbf{r}_{j}\right)\right\rangle
$$

is the particle number density distribution function and $m(\mathbf{r}, \hat{R})$ is the orientational distribution function at position $\mathbf{r}$. From Eqs. (10), (22), and (23), it follows that

$$
n(\mathbf{r})=\int d \hat{R} \rho(\mathbf{r}, \hat{R})
$$

and

$$
\int d \hat{R} m(\mathbf{r}, \hat{R})=1
$$

When Eq. (22) is introduced, Eq. (11) becomes

$$
\begin{aligned}
\Omega[n, m]= & k_{B} T \int d 1 n\left(r_{1}\right) m(1) \log m(1)+\int d \mathbf{r}_{1} f^{d}\left(n\left(r_{1}\right)\right) \\
& -\int d 1 n\left(r_{1}\right) m(1)[\mu-v(1)] \\
& +\frac{1}{2} \iint d \mathbf{r}_{1} d \mathbf{r}_{2} n\left(r_{1}\right) n\left(r_{2}\right) H\left(r_{12}-d\right) \phi^{\text {att }}\left({ }_{12}\right) \\
& +\frac{1}{2} \iint d 1 d 2 n\left(r_{1}\right) n\left(r_{2}\right) m(1) m(2) \\
& \times H\left(r_{12}-d\right) \phi^{\mathrm{mp}}(1,2),
\end{aligned}
$$

where $f^{d}(n)$ is the Helmholtz free energy density per unit volume of the hard sphere fluid, and is given by

$$
f^{d}(n)=k_{B} \operatorname{Tn}\left[\log \left(\Lambda^{3} n\right)-1\right]+f^{\mathrm{exc}}(n) .
$$

Since $U(1)$ obtained in Eq. (19) is now a one body potential, we make an ansatz:

$$
m(1)=\frac{1}{Z_{R}\left(r_{1}\right)} \exp \left\{-\frac{U(1)}{k_{B} T}\right\},
$$

which is the orientational distribution, in an external field $U(1)$, of a molecule that is otherwise isolated. $Z_{R}\left(r_{1}\right)$ is a normalization constant required by Eq. (25)

$$
Z_{R}\left(r_{1}\right)=\int d \hat{R} \exp \left\{-\frac{U(1)}{k_{B} T}\right\} .
$$

Equation (28) shows that we can introduce $U(1)$ as a new order parameter in place of $m(1)$. An alternative, yet physically more transparent, choice is $q^{\text {eff }}\left(r_{1}\right)$, which is related to the local dielectric constant $\epsilon\left(r_{1}\right)$ through Eq. (18). For arbitrary functions $G_{a}(1)$ and $G_{b}(1,2)$, we define their angular averages by

$$
\left\langle G_{a}(1)\right\rangle_{\hat{R}_{1}} \equiv \int d \hat{R}_{1} m(1) G_{a}(1)
$$

and

$$
\left\langle G_{b}(1,2)\right\rangle_{\hat{R}_{1} \hat{R}_{2}} \equiv \iint d \hat{R}_{1} d \hat{R}_{2} m(1) m(2) G_{b}(1,2),
$$

respectively. Using Eqs. (14), (19), and (28), we rewrite Eq. (26) as follows:

$$
\begin{aligned}
\Omega\left[n, q^{\mathrm{eff}}\right]= & \int d \mathbf{r}_{1} f^{d}\left(n\left(r_{1}\right)\right)-\int d \mathbf{r}_{1} n\left(r_{1}\right)\left\{\mu+k_{B} T \log \left[Z_{R}\left(r_{1}\right)\right]\right\}+\int d \mathbf{r}_{1} n\left(r_{1}\right)\left[q^{\mathrm{ion}}-q^{\mathrm{eff}}\left(r_{1}\right)\right]\left\langle u^{T}(1)\right\rangle_{\hat{R}_{1}} \\
& +\frac{1}{2} \iint d \mathbf{r}_{1} d \mathbf{r}_{2} n\left(r_{1}\right) n\left(r_{2}\right) H\left(r_{12}-d\right) \phi^{\mathrm{att}}\left(r_{12}\right)+\frac{1}{2} \iint d \mathbf{r}_{1} d \mathbf{r}_{2} n\left(r_{1}\right) n\left(r_{2}\right) H\left(r_{12}-d\right)\left\langle\phi^{\mathrm{mp}}(1,2)\right\rangle_{\hat{R}_{1} \hat{R}_{2}} .
\end{aligned}
$$

We have replaced $v(1)$ by $U^{\mathrm{pol}}(1)+U^{\mathrm{ion}}(1)$. The hard core repulsion of the ion imposes the boundary condition

$$
n(r)=0 \quad\left(\text { if } r<r^{\text {ion }}+d / 2\right) .
$$

As before, the stationarity condition of $\Omega$ determines the equilibrium profile for $n(r)$ and $q^{\text {eff }}(r)$ :

$$
\frac{\delta \Omega}{\delta n}=0 \quad \text { and } \quad \frac{\delta \Omega}{\delta q^{\text {eff }}}=0 .
$$

Noting that $q^{\text {eff }}\left(r_{1}\right)$ dependence of $\Omega$ is explicit in the third term of Eq. (32) and also implicit in $Z_{R}\left(r_{1}\right)$ and the angular 
averages indicated by $\langle\cdots\rangle_{\hat{R}_{1}}$ and $\langle\cdots\rangle_{\hat{R}_{1} \hat{R}_{2}}$, Eq. (34) becomes

$$
\begin{aligned}
0= & \mu^{d}\left(n\left(r_{1}\right)\right)-\left\{\mu+k_{B} T \log \left[Z_{R}\left(r_{1}\right)\right]\right\}+\left[q^{\mathrm{ion}}-q^{\mathrm{eff}}\left(r_{1}\right)\right] \\
& \times\left\langle u^{T}(1)\right\rangle_{\hat{R}_{1}}+\int d \mathbf{r}_{2} n\left(r_{2}\right) H\left(r_{12}-d\right) \phi^{\mathrm{att}}\left(r_{12}\right) \\
& +\int d \mathbf{r}_{2} n\left(r_{2}\right) H\left(r_{12}-d\right)\left\langle\phi^{\mathrm{mp}}(1,2)\right\rangle_{\hat{R}_{1} \hat{R}_{2}}
\end{aligned}
$$

and

$$
\begin{aligned}
0= & {\left[q^{\mathrm{eff}}\left(r_{1}\right)-q^{\mathrm{ion}}\right]\left\{\left[\left\langle u^{T}(1)^{2}\right\rangle_{\hat{R}_{1}}-\left\langle u^{T}(1)\right\rangle_{\hat{R}_{1}}^{2}\right]\right.} \\
& \left.+k_{B} T\left\langle u^{\mathrm{pol}}(1)\right\rangle_{\hat{R}_{1}}\right\}-\int d \mathbf{r}_{2} n\left(r_{2}\right) H\left(r_{12}-d\right) \\
& \times\left\{\left[\left\langle\phi^{\mathrm{mp}}(1,2) u^{T}(1)\right\rangle_{\hat{R}_{1} \hat{R}_{2}}-\left\langle\phi^{\mathrm{mp}}(1,2)\right\rangle_{\hat{R}_{1} \hat{R}_{2}}\left\langle u^{T}(1)\right\rangle_{\hat{R}_{1}}\right]\right. \\
& \left.-k_{B} T\left\langle\frac{\partial \phi^{\mathrm{mp}}(1,2)}{\partial q^{\mathrm{eff}}\left(r_{1}\right)}\right\rangle_{\hat{R}_{1} \hat{R}_{2}}\right\},
\end{aligned}
$$

where $\mu^{d}(n) \equiv \partial f^{d} / \partial n$. The derivation of Eq. (36) is rather lengthy and is given in Appendix D.

Equations (32), (35), and (36) constitute the central result at this stage. Briefly, first we solve Eqs. (35) and (36) to obtain the equilibrium profiles of $n(r)$ and $q^{\text {eff }}(r)$ in $r>r^{\text {ion }}+d / 2$. The obtained profiles are substituted into Eq. (32) to evaluate the grand potential of the system. As shown in Appendix E, some of the integrations indicated in these equations are analytically tractable, reducing the dimensionality of the integrals in Eq. (32) to at most four and those in Eqs. (35) and (36) to at most three.

\section{Bulk properties}

In the absence of the external field, the thermodynamic properties of a homogeneous system can be easily derived from Eq. (32). Let $q^{\text {ion }}=0$, then Eq. (36) has a trivial solution of $q^{\text {eff }}(r)=0$. In fact, if $q^{\text {eff }}(r)=0, U(1)$ given by Eq. (19) becomes zero and hence $m(1)$ is constant. Then the angular averages of the total multipole moments on the molecules 1 and 2 are all zero, for these angular averaged tensors are spherically symmetric as well as traceless. Since $\phi^{\mathrm{mp}}(1,2)$ is a sum of the terms proportional to the total multipole moments of the molecules 1 and 2 , the terms involving $\phi^{\mathrm{mp}}(1,2)$ in Eq. (36) are zero and Eq. (36) is identically satisfied. This also means that under the mean field approximation employed in the present work, bulk properties of the system are, in the absence of the external field, the same as those of the system of molecules without polarizabilities or permanent multipole moments.

Setting $n(r)$ to a constant $n$ in Eq. (32), we obtain the Helmholtz free energy density:

$$
\begin{aligned}
f(T, n) & =-p+\mu n \\
& =-k_{B} T n \log f_{R}+f^{d}(n)-\frac{1}{2} \alpha^{\mathrm{att}} n^{2} .
\end{aligned}
$$

Note that $\Omega=-p V$ for a homogeneous system. $f_{R}$ is the contribution from the free rotation of a molecule, the value of which is $8 \pi^{2}$ in general and equals $4 \pi$ for a linear molecule. $\alpha^{\text {att }}$ is defined here by

$$
\begin{aligned}
\alpha^{\mathrm{att}} & \equiv-\frac{1}{V} \iint d \mathbf{r}_{1} d \mathbf{r}_{2} H\left(r_{12}-d\right) \phi^{\mathrm{att}}\left(r_{12}\right) \\
& =-\int d \mathbf{r}_{12} H\left(r_{12}-d\right) \phi^{\mathrm{att}}\left(r_{12}\right) .
\end{aligned}
$$

For the particular choice of $\phi^{\text {att }}\left(r_{12}\right)$ given by Eq. (3), one has

$$
\alpha^{\mathrm{att}}=\frac{4 \pi}{3} \epsilon^{\mathrm{att}} d^{3} .
$$

Equation (37) is a fundamental equation of the isotropic system. Note that Eq. (37) is essentially the same as for the isotropic system of molecules without polarizability or permanent multipole moments. $f_{R}$ merely affects the value of $f(T, n)$ at the standard state. From Eq. (37) by well known thermodynamic relations, one obtains

$$
\begin{aligned}
& \mu(T, n)=-k_{B} T \log f_{R}+\mu^{d}(n)-\alpha^{\text {att }} n, \\
& p(T, n)=n \mu^{d}(n)-f^{d}(T, n)-\frac{1}{2} \alpha^{\text {att }} n^{2} .
\end{aligned}
$$

At a given temperature, the coexisting bulk densities are determined by

$$
\begin{aligned}
& \mu_{l}\left(T, n_{l}^{\mathrm{eq}}\right)=\mu_{v}\left(T, n_{v}^{\mathrm{eq}}\right), \\
& p_{l}\left(T, n_{l}^{\mathrm{eq}}\right)=p_{v}\left(T, n_{v}^{\mathrm{eq}}\right),
\end{aligned}
$$

where the subscripts $l$ and $v$ refer to liquid and vapor, respectively. The spinodal curve which divides the metastable and unstable regions in $T-n$ phase diagram is obtained by

$$
\frac{\partial p}{\partial n}=0 .
$$

The critical point is located in the phase diagram by Eq. (43) and

$$
\frac{\partial^{2} p}{\partial n^{2}}=0,
$$

with a numerical solution

$$
\begin{aligned}
& y_{c}=\frac{\pi}{6} d^{3} n_{c} \\
& \approx 0.13044 ; \\
& \frac{1}{k_{B} T_{c}} \approx 2.6503\left(\frac{1}{\epsilon^{\mathrm{att}}}\right) .
\end{aligned}
$$

\section{SOLUTION METHODS FOR THE EQUILIBRIUM PROFILES}

Given $T / T_{c}$ and supersaturation $S$, defined as the ratio of the metastable vapor pressure to the equilibrium vapor pressure, we can calculate, via Eqs. (40)-(42), the chemical potential $\mu$ of the system and the densities of the bulk liquid $n_{l}$ and vapor $n_{v}$ at that chemical potential. For thus obtained $\mu$ 
and $n_{v}$, Eqs. (35) and (36) have to be solved simultaneously with the boundary conditions given by Eq. (33) and

$$
n(r) \rightarrow n_{v} \quad \text { as } \quad r \rightarrow \infty .
$$

Let $\delta n(r)$ and $\delta q^{\text {eff }}(r)$ be the deviations of $n(r)$ and $q^{\text {eff }}(r)$, respectively, from the exact solutions of Eqs. (35) and (36). Expanding $\Omega\left[n, q^{\text {eff }}\right]$ around the approximate profiles and ignoring the higher order, one finds that the error in $\Omega$, which we denote by $\Delta \Omega$, due to the deviations of $n(r)$ and $q^{\text {eff }}(r)$ from the exact solutions, satisfies

$$
\begin{aligned}
|\Delta \Omega| & \leqslant \int d \mathbf{r}_{1}\left|\frac{\delta \Omega}{\delta n\left(r_{1}\right)} \delta n\left(r_{1}\right)\right|+\int d \mathbf{r}_{1}\left|\frac{\delta \Omega}{\delta q^{\mathrm{eff}}\left(r_{1}\right)} \delta q^{\mathrm{eff}}\left(r_{1}\right)\right| \\
& \leqslant n_{\max } \int d \mathbf{r}_{1}\left|\frac{\delta \Omega}{\delta n\left(r_{1}\right)}\right|+\left|q^{\mathrm{ion}}\right| \int d \mathbf{r}_{1}\left|\frac{\delta \Omega}{\delta q^{\mathrm{eff}}\left(r_{1}\right)}\right|
\end{aligned}
$$

where $n_{\max }=\sqrt{2} / d^{3}$ is the density at the closest packing. Note that the rescaled charge $q^{\text {eff }}\left(r_{1}\right)$ never exceeds $q^{\text {ion }}$. We define

$$
\begin{aligned}
& \Delta_{n} \equiv n_{\max } \int d \mathbf{r}_{1}\left|\frac{\delta \Omega}{\delta n\left(r_{1}\right)}\right|, \\
& \Delta_{q} \equiv\left|q^{\text {ion }}\right| \int d \mathbf{r}_{1}\left|\frac{\delta \Omega}{\delta q^{\text {eff }}\left(r_{1}\right)}\right|,
\end{aligned}
$$

and demand that both $\Delta_{n}$ and $\Delta_{q}$ be sufficiently small.

Under a condition that nucleation takes place, Eqs. (35) and (36) have two sets of solutions for $n(r)$ and $q^{\text {eff }}(r)$. One is for a metastable state exhibiting the vapor solvation of an ion and the other is for a critical nucleus, which corresponds to a saddle point in the functional space. The metastable profiles for $n(r)$ and $q^{\text {eff }}(r)$ are obtained by iteration. In particular, we start from the initial guess

$$
q^{\text {eff }}(r)=q^{\text {ion }} \quad \text { and } \quad n(r)=n_{v} .
$$

For this $n(r)$, we can solve Eq. (36) by iteration until $\Delta_{q}$ becomes sufficiently small. The resulting $q^{\text {eff }}(r)$ is used in Eq. (35), which is now iterated just once. Using $n(r)$ and $q^{\text {eff }}(r)$ thus obtained as the next guess, we repeat the same procedure until both $\Delta_{n}$ and $\Delta_{q}$ become sufficiently small. To obtain the critical nucleus by iteration, we take

$$
q^{\text {eff }}(r)=q^{\text {ion }} \quad \text { and } \quad n(r)= \begin{cases}n_{l} & r \leqslant R \\ n_{v} & \text { otherwise }\end{cases}
$$

as the initial guess and proceed in the same manner as for the metastable nucleus. If $R$ is too small, the nucleus shrinks as the iteration proceeds, while it grows if $R$ is too large. Starting from several values of $R$, it is possible to find $R^{*}$ such that the nucleus neither shrinks nor grows as the iteration is repeated. In the actual computation, $R^{*}$ was identified with that which yields, after some steps of iteration, $n(r)$ that minimizes $\Delta_{n}$. Then, this $n(r)$ was used with the corresponding $q^{\text {eff }}\left(r_{1}\right)$ as the initial guess in solving Eq. (35) more accurately by the Newton-Raphson method, after which the iterative solution of Eq. (36) follows. Using $n(r)$ and $q^{\text {eff }}(r)$ thus obtained as the next guess, we repeatedly applied the process until both $\Delta_{n}$ and $\Delta_{q}$ become sufficiently small. The grand potential $\Omega$ of a system was calculated from Eq. (32) for the obtained equilibrium profiles. The reversible work of nucleation $\Delta \Omega$, not to be confused with that in Eq. (47), was calculated as the difference of $\Omega$ for the critical nucleus and for the metastable nucleus.

Finally, the boundary of the system is taken to be a sphere, the radius $r_{0}$ of which is sufficiently large so that $n\left(r_{0}\right)$ and $q^{\text {eff }}\left(r_{0}\right)$ attain their limiting values $n_{v}$ and $q^{\text {ion }} / \epsilon_{v}$. Here $\epsilon_{v}$ is the dielectric constant of the bulk vapor of density $n_{v}$. There are two contributions to the free energy density at $r_{1}<r_{0}$ resulting from the interaction across the system boundary at $r_{0}$. One is through $\phi^{\text {att }}\left(r_{12}\right)$ and can be evaluated analytically. The other is through $\phi^{\mathrm{mp}}(1,2)$, which was calculated numerically by noting that the contribution to the free energy density at $r_{1}$ comes from only those molecules in the spherical shell of $r_{L}<r<r_{1}+d$, where $r_{L}$ is the larger of $r^{\text {ion }}+d / 2$ and $r_{1}-d$. (See Appendix E.) The free energy den-

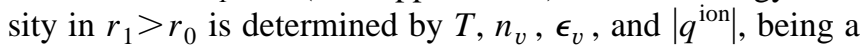
constant which is same for the critical nucleus and for the metastable nucleus. This allows one to calculate the free energy difference between two systems which have the same $T$, $n_{v}, \epsilon_{v}$, and $\left|q^{\text {ion }}\right|$ without introducing any truncation error.

\section{RESULTS AND THEIR IMPLICATIONS}

In applying the above density functional theory to a particular substance, one needs to know its critical temperature $T_{c}$, the molecular diameter $d$, the polarizabilities, and the permanent multipole moments. The value of $T_{c}$ is generally available ${ }^{20}$ and $d$ can be estimated from the molecular geometry. The values of the polarizabilities and the permanent multipole moments can be obtained from quantum mechanical calculations. The results are available for some materials, e.g., $\left(\mathrm{CH}_{3}\right)_{2} \mathrm{O}, \mathrm{CH}_{3} \mathrm{OH}, \mathrm{CH}_{4}, \mathrm{CS}_{2}$, and $\mathrm{H}_{2} \mathrm{O}$. ${ }^{21-23}$ Among these, perhaps the most interesting case would be $\mathrm{H}_{2} \mathrm{O}$. However, any sensible treatment of water requires a proper account of hydrogen bonding and is not attempted in the current work. We have limited our application to ion-induced nucleation of $\mathrm{CS}_{2}, \mathrm{CH}_{4}$, and $\mathrm{CCl}_{4}$. Except for $\mathrm{CCl}_{4}$, these materials are rarely used in experiments, yet they are highly symmetric, thereby reducing the computational work, while still serving to illustrate some of the essential features of ion-induced nucleation. The values of the molecular parameters used in this work are given in Table I.

We nondimensionalized relevant quantities by model parameters: $d$ as the length scale, $k_{B} T_{c}$ as the energy scale, and $|e|$ for electric charge, where $e$ is the electron charge. Nondimensionalized quantities are denoted by $\sim$ (tilde).

\section{A. $\mathrm{CS}_{2}$}

Figure 2 shows the density profile $n(r)$ of $\mathrm{CS}_{2}$ obtained at $\tilde{T}=0.55$ and supersaturation $S=2$. As mentioned in Sec. III, a metastable profile shows solvation of the ion. Volume exclusion due to the ion surface, which is regarded as a hard wall, is apparent outside the first coordination shell. The general feature of particular interest is that near the ion surface the number density $n(r)$ is higher when $q^{\text {ion }}<0$ both for the metastable and for the critical nucleus than when $q^{\text {ion }}>0$. This indicates that the ion-molecule interaction is stronger if $q_{\text {ion }}<0$, implying some sort of asymmetry in the interaction. 
TABLE I. Material constants for $\mathrm{CS}_{2}$ and $\mathrm{CH}_{4}$ (Refs. 22 and 23). $a_{0}$ and $e$ are Bohr radius and the electron charge, respectively. As indicated by the superscript $(B)$, tensor components are expressed in body fixed coordinate system (Refs. 22 and 23). Values of the other tensor components are readily deduced from those given here from the molecular symmetry. The $\mathrm{C}-\mathrm{Cl}$ bond length is taken from Ref. 24, while the values of the critical temperature are taken from Ref. 20. $b$ is defined in Eq. (54). The values of the polarizabilities and the permanent multipole moments for $\mathrm{CCl}_{4}$ are estimated from the corresponding values for $\mathrm{CH}_{4}$ as explained in Sec. IV B.

\begin{tabular}{lccc}
\hline \hline & $\mathrm{CS}_{2}$ & $\mathrm{CH}_{4}$ & $\mathrm{CCl}_{4}$ \\
\hline$T_{c}$ & $552 \mathrm{~K}$ & $190.5 \mathrm{~K}$ & $556.6 \mathrm{~K}$ \\
$d$ & $\sim 5.868 a_{0}$ & $\sim 4.104 a_{0}$ & $\sim 6.654 a_{0}$ \\
& $(\mathrm{~S}-\mathrm{S}$ distance $)$ & $(2 \times \mathrm{C}-\mathrm{H}$ distance $)$ & $(2 \times \mathrm{C}-\mathrm{Cl}$ distance $)$ \\
$b$ & 97.62 & 404.4 & 85.40 \\
$\mu$ & 0 & 0 & 0 \\
$\Theta$ & $\Theta_{33}^{(B)}=2.425|e| a_{0}{ }^{2}$ & 0 & 0 \\
$\Omega$ & 0 & $\Omega_{123}^{(B)}=2.410|e| a_{0}{ }^{3}$ & $\Omega_{123}^{(B)}=-10.98|e| a_{0}{ }^{3}$ \\
$\Phi$ & $\Phi_{3333}^{(B)}=140.1|e| a_{0}{ }^{4}$ & $\Phi_{3333}^{(B)}=-7.690|e| a_{0}{ }^{4}$ & $\Phi_{3333}^{(B)}=58.07|e| a_{0}{ }^{4}$ \\
$\alpha$ & $\alpha_{11}^{(B)}=\alpha_{22}^{(B)}$ & $\alpha_{33}^{(B)}=15.98 a_{0}{ }^{3}$ & $\alpha_{33}^{(B)}=72.80 a_{0}{ }^{3}$ \\
& $=36.58 a_{0}{ }^{3}$ & & \\
& $\alpha_{33}^{(B)}=93.14 a_{0}{ }^{3}$ & & \\
$A$ & 0 & $A_{1,23}^{(B)}=9.46 a_{0}{ }^{4}$ & $A_{1,23}^{(B)}=71.44 a_{0}{ }^{4}$ \\
\hline \hline
\end{tabular}

To see this more explicitly, consider the bare ion-molecule interaction energy $U^{b}(1)$, which is obtained by setting $q^{\text {eff }}\left(r_{1}\right)=q^{\text {ion }}$ in Eqs. (19) $-(21)$ :

$$
U^{b}(1)=-\frac{1}{2} q^{\mathrm{ion}^{2}} u^{\mathrm{pol}}(1)+q^{\mathrm{ion}} u^{P}(1) .
$$

Since $\mathrm{CS}_{2}$ is a linear centro-symmetric molecule, $U^{b}(1)$ depends only on the ion-molecule separation $r_{1}$ and the angle $\theta_{1}$ between the radial direction $\mathbf{r}_{1}$ and $\mathrm{C}-\mathrm{S}$ bond. We plotted $U^{b}(1)$ in Fig. 3, as a function of $\left|\cos \theta_{1}\right|$ at $r_{1}=1.5 d$, namely, at the minimum ion-molecule separation when $r^{\text {ion }}=d$. The sign dependence of $U^{b}(1)$ shown in Fig. 3 can be readily understood as follows. As shown in Fig. 4, the first term in Eq. (51) is minimum when the $\mathrm{C}-\mathrm{S}$ bond lines up with the electric field due to $q^{\text {ion }}$, irrespective of its sign, while the second term yields the minimum value at the angle which changes from $\left|\cos \theta_{1}\right|=1$ to $\left|\cos \theta_{1}\right| \approx 0.45$ as $q^{\text {ion }}$ changes its sign from negative to positive. Stated differently, when $q^{\text {ion }}<0$, the polarizabilities and the permanent multipole moments work constructively to orient the molecule along the direction of the electric field while they work rather destructively if $q^{\text {ion }}>0$.

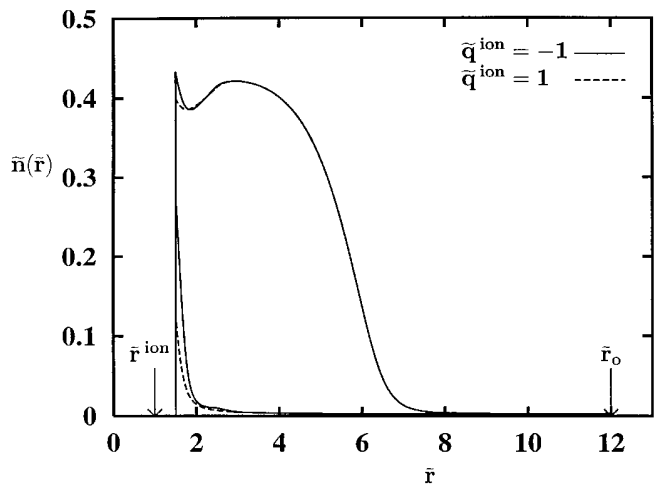

FIG. 2. Equilibrium density profiles of $\mathrm{CS}_{2}$ at $\tilde{T}=0.55$ and $S=2$. For each value of $\tilde{q}^{\text {ion }}$, the lower profile corresponds to the metastable nuclei, while the upper profile represents the critical nuclei.
The sign dependence of $U^{b}(1)$ is present for a molecule even with only a single permanent multipole moment, higher than the dipole, without polarizabilities. Thus in the case of a linear centro-symmetric molecule, the interaction energy between the ion (point charge) and the quadrupole moment of the molecule depends quadratically on $\cos \theta_{1}$, attaining the minimum value at $\cos \theta_{1}=0$ for one sign of the ion charge which is, in general, different from what is obtained at $\cos \theta_{1}= \pm 1$ as the minimum for the other sign of the ion charge. Such possibility of producing the sign effect from a single permanent multipole moment is not pursued here, for the contribution to $U^{b}(1)$ from each one of the permanent multipole moments or the polarizabilities is comparable to each other at least for those molecules close to the ion.

The sign dependence of $U^{b}(1)$ is counteracted to some extent by the dielectric response of the condensing molecules. Figure 5 shows the variation of the local dielectric constant $\epsilon(r)$ corresponding to the density profile $n(r)$ shown in Fig. 2. For a given value of $r$, a profile with a smaller value of $\epsilon(r)$ applies for the metastable nucleus while the larger corresponds to the critical nucleus. Clearly,

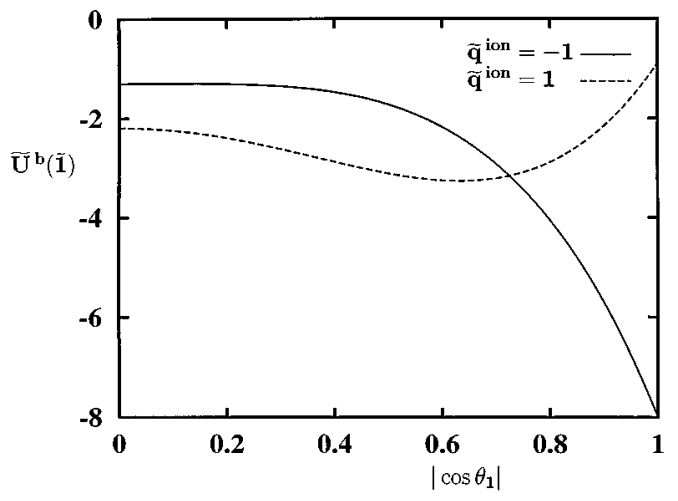

FIG. 3. The bare ion-molecule interaction potential $\tilde{U}^{b}(\tilde{1})$ for $\mathrm{CS}_{2}$ at $\tilde{r}_{1}=1.5$ and $\left|\tilde{q}^{\text {ion }}\right|=1$. 


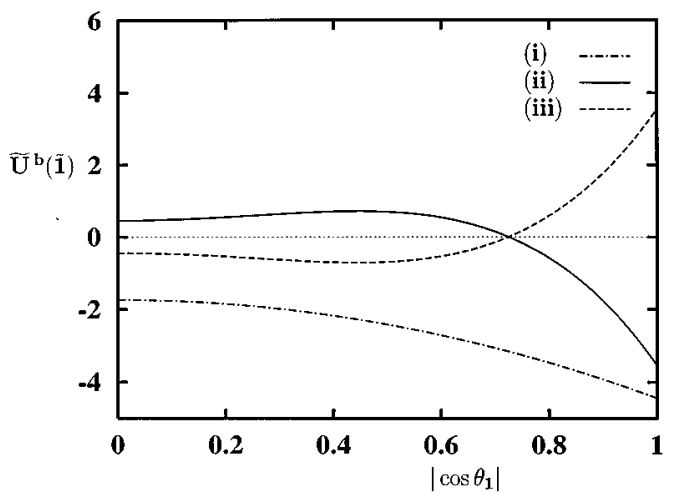

FIG. 4. Contributions for $\tilde{U}^{b}(\tilde{1})$ from (i) the polarizabilities $\left[-\frac{1}{2} q^{\text {ion }^{2}} u^{\mathrm{pol}}(1) / k_{B} T_{c}\right]$ and the permanent multipole moments $\left[q^{\text {ion }} u^{P}(1) / k_{B} T_{c}\right]$ with (ii) $\tilde{q}^{\text {ion }}=-1$ and (iii) $\tilde{q}^{\text {ion }}=1$.

the effective electric field around the ion is weaker when $q^{\text {ion }}<0$ compared to the case of $q^{\text {ion }}>0$. Recall the definition of $\epsilon(r)$ given in Eq. (18).

Density profiles of $\mathrm{CS}_{2}$ in the case of $\tilde{q}_{\text {ion }}=-1$ are shown in Fig. 6 at $\tilde{T}=0.55$ for several values of the supersaturation. Figure 6 shows a similar feature to that observed in our previous work on ion-induced nucleation of a dipolar fluid. ${ }^{15}$ Namely, a metastable nucleus grows as $S$ increases, while the critical nucleus shrinks. These two profiles eventually coincide at a certain supersaturation $S<S_{\max }$, where $S_{\max }$ is the supersaturation at the spinodal, indicating the onset of instability of the vapor phase in the presence of the ion. These trends can be explained by a completely parallel argument to that given previously ${ }^{15}$ and will not be repeated here.

Figure 7 shows the local dielectric constant profile $\epsilon(r)$ under the same condition as for Fig. 6. Comparing Fig. 2 with Fig. 5 and Fig. 6 with Fig. 7, one finds that $\epsilon(r)$ at $r$ is roughly proportional to the local density $n(r)$ at that point. A remarkable exception is the sharp peak in $\epsilon(r)$ near the ion surface and the oscillation following the peak; the latter is particularly clear for a large nucleus. To understand this behavior, we obtained $\epsilon(r)$ at $\tilde{T}=0.55$ corresponding to a sim-

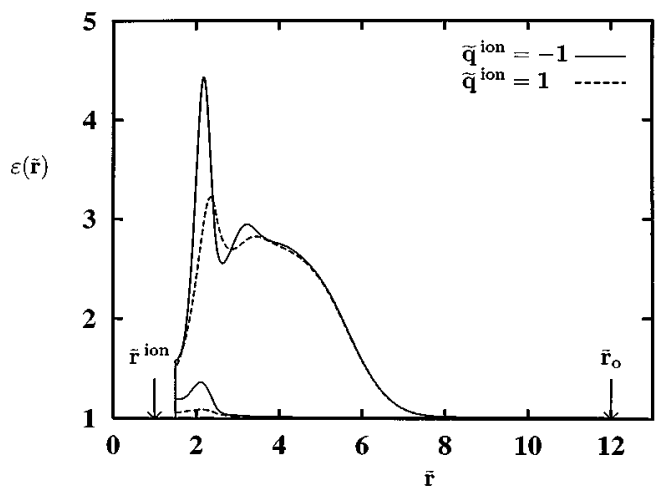

FIG. 5. Variation of the local dielectric constant $\epsilon(\tilde{r})$ corresponding to the density profile $\tilde{n}(\tilde{r})$ given in Fig. 2 . For each value of $\tilde{q}^{\text {ion }}$, the lower profile corresponds to the metastable nuclei, while the upper profile represents the critical nuclei.

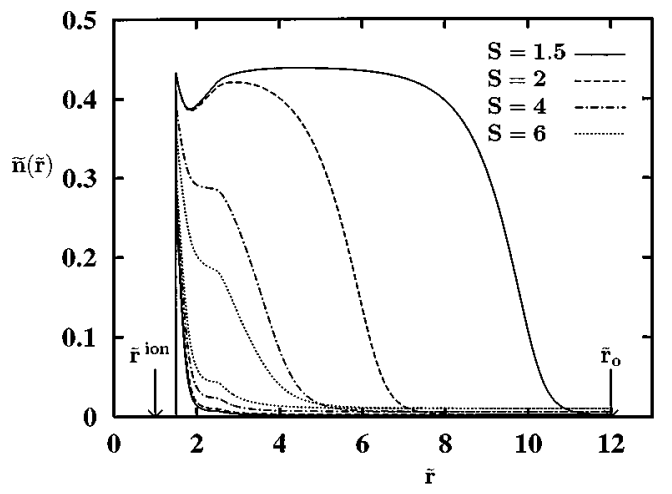

FIG. 6. Variation in the density profiles $\tilde{n}(\tilde{r})$ of $\mathrm{CS}_{2}$ with the supersaturation $S . \tilde{T}=0.55$ and $\tilde{q}^{\text {ion }}=-1$.

pler density profile $n(r)=n_{\max }$. Figure 8 shows that the sharp peak followed by the oscillation persists even if $n(r)$ is constant throughout. As shown in Appendix E, only those molecules in the spherical shell $r_{L}<r<r_{1}+d$ contribute to the dielectric constant $\epsilon\left(r_{1}\right)$ at $r_{1}$. In the present case of uniform density, the number of molecules $N_{\epsilon}\left(r_{1}\right)$ in this spherical shell is proportional to the volume of the shell and a monotonically increasing function of $r_{1}$. On the other hand, as shown in Appendix E, contributions to the integral in Eq. (36) tend to cancel and the average total multipole moments on a molecule decrease as $r_{1}$ is increased, both of which reduce the magnitude of the integral in Eq. (36). At the $r_{1} \rightarrow \infty$ limit, the increase in $N_{\epsilon}\left(r_{1}\right)$ balances exactly the decrease in the magnitude of the integral to yield the constant value of $\epsilon(\infty)$. However, when $r_{1}<r^{\text {ion }}+\frac{3}{2} d$, the increase in $N_{\epsilon}\left(r_{1}\right)$ is due partially to the increase in the spherical shell thickness, which leads to the steep increase in $\epsilon\left(r_{1}\right)$ in this range of $r_{1}$. Now, the existence of a peak implies that there must be a competing factor with this steep increase in $\epsilon\left(r_{1}\right)$. To see how this happens, first recall that $\epsilon\left(r_{1}\right)$ is essentially the reciprocal of the effective electric field due to $q^{\text {ion }}$ and other molecules in $r_{L}<r_{2}<r_{1}+d$, the field by the latter being in the opposite direction to the former. When $\epsilon\left(r_{2}\right)$ is larger, the electric field at $r_{1}$ created by those molecules in this region is smaller, causing the decrease in $\epsilon\left(r_{1}\right)$.

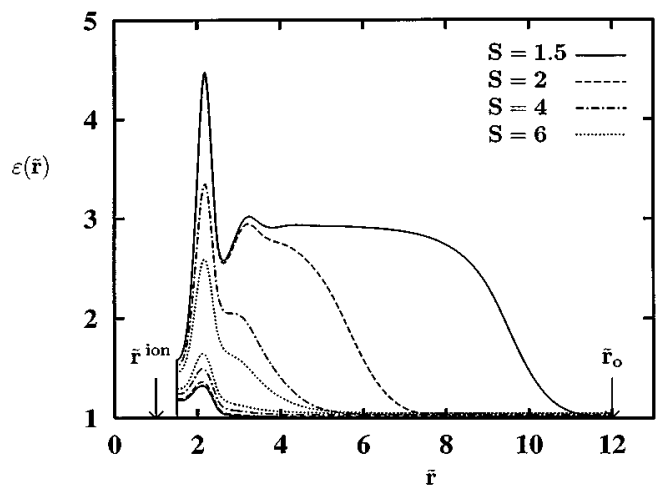

FIG. 7. Local dielectric constants $\epsilon(\tilde{r})$ corresponding to the density profiles shown in Fig. 6. 


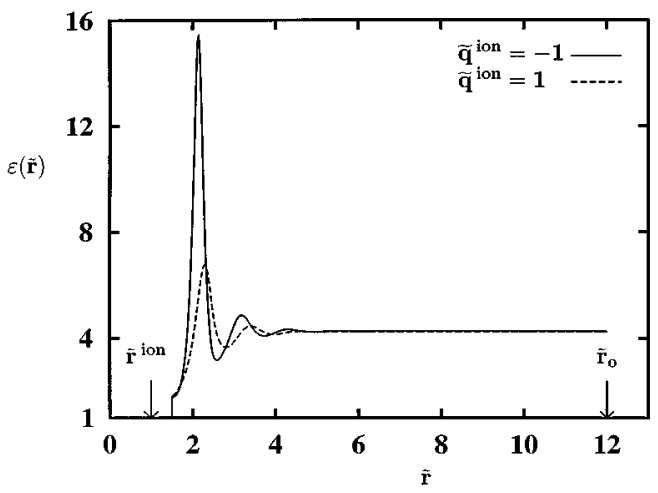

FIG. 8. Local dielectric constants $\epsilon(\tilde{r})$ at $\tilde{T}=0.55$ corresponding to the density profile $n(r)=n_{\max }$.

A peak in $\epsilon(r)$ near the ion arises as a result of these two competing factors. The same mechanism is responsible for the oscillation of $\epsilon(r)$ mentioned above.

Figure 9 shows the difference between the reversible work $\Delta \Omega_{+} / k_{B} T$ of nucleation of $\mathrm{CS}_{2}$ on a positive ion and that $\left(\Delta \Omega_{-} / k_{B} T\right)$ on a negative ion. Calculations were performed close to but below the supersaturation at which the metastable nucleus reaches its stability limit. For both cases, this happens at $S<S_{\max }$, but first for the case of $q^{\text {ion }}<0$ as the result of the preference to the negative ion shown by $U^{b}(1)$. Strictly speaking, one has to take into account the effect of the dielectric response of the condensing molecules. In all of our calculations for $\mathrm{CS}_{2}$, however, we found that the quantities defined as

$$
\begin{aligned}
& \Delta \Omega^{\text {critical }} \equiv \Omega_{+}^{\text {critical }}-\Omega_{-}^{\text {critical }}, \\
& \Delta \Omega^{\text {metastable }} \equiv \Omega_{+}^{\text {metastable }}-\Omega_{-}^{\text {metastable }}
\end{aligned}
$$

were both positive, being consistent with the sign preference of $U^{b}(1)$. Here $\Omega^{\text {critical }}$ and $\Omega^{\text {metastable }}$, respectively, denote the grand potential of a system with a critical nucleus and a metastable nucleus. We see that the observed sign preference of the reversible work is also consistent with that shown by $U^{b}(1)$. As we shall see below, however, the latter is not

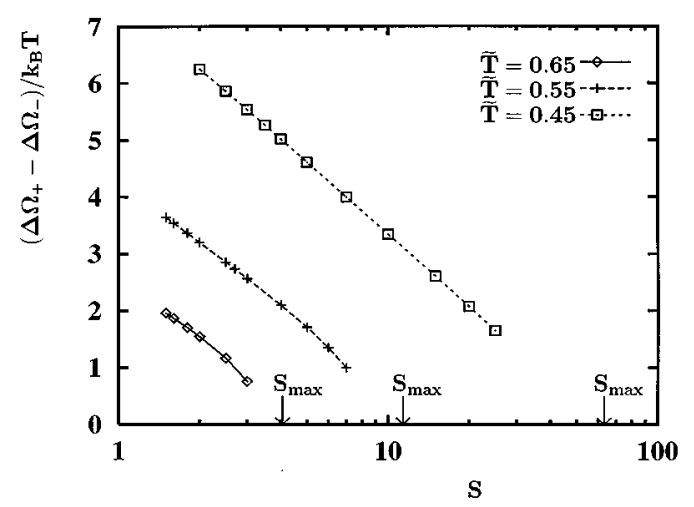

FIG. 9. The difference in the reversible work of nucleation of $\mathrm{CS}_{2}$ between the cases of $\tilde{q}^{\text {ion }}=1$ and $\tilde{q}^{\text {ion }}=-1$. $\tilde{r}^{\text {ion }}=1$. Three values of $S_{\max }$ represent the supersaturation at the spinodal. From the left, they correspond to $\tilde{T}=0.65$, $\tilde{T}=0.55$, and $\tilde{T}=0.45$, respectively.

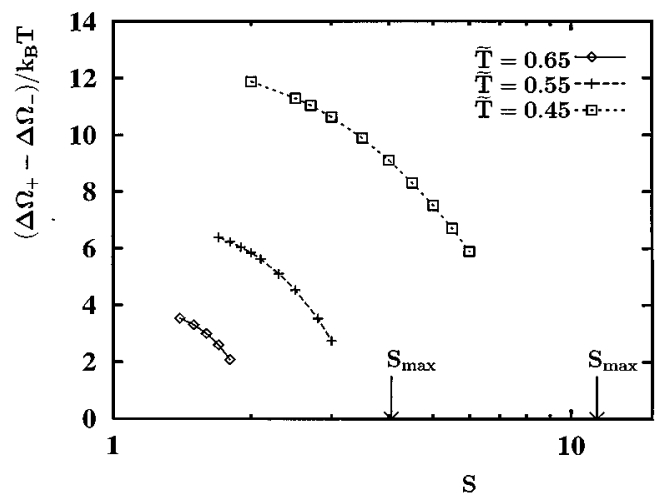

FIG. 10. Same as Fig. 9, but for $\mathrm{CH}_{4} . S_{\max }$ for $\tilde{T}=0.45$ is not shown in the figure.

always the case. A similar monotonic decrease in $\Delta \Omega_{+}-\Delta \Omega_{-}$with increasing $S$ is observed to that found previously for dipolar molecules. ${ }^{15}$

\section{B. $\mathrm{CH}_{4}$ and $\mathrm{CCl}_{4}$}

Figure 10 shows the sign preference in the case of $\mathrm{CH}_{4}$. A similar trend is found to the case of $\mathrm{CS}_{2}$. In Fig. 11, we show the sign preference of the reversible work for $\mathrm{CH}_{4}$ and $\mathrm{CCl}_{4}$. In both calculations, $\tilde{T}=0.55$ and $r^{\text {ion }}$ is set equal to the diameter of a $\mathrm{CH}_{4}$ molecule.

The calculation for $\mathrm{CCl}_{4}$ is only qualitative. Since not all of the required molecular parameters are available for this molecule, we estimated them from the corresponding values for $\mathrm{CH}_{4}$. We simply assumed that the polarizabilities and the permanent multipole moments scale with proper powers of the molecular size and that the permanent multipole moments have a sign opposite to those of $\mathrm{CH}_{4}$. The latter assumption follows from the relative electronegativites: ${ }^{24} \mathrm{Cl}$ $>\mathrm{C}>\mathrm{H}$.

Here again, calculations are made close to but below the supersaturation at which a metastable nucleus becomes unstable. From Fig. 11, it is observed that the ion is more effective for $\mathrm{CH}_{4}$ both in reducing the nucleation barrier and in producing the sign preference. When nondimensionalized,

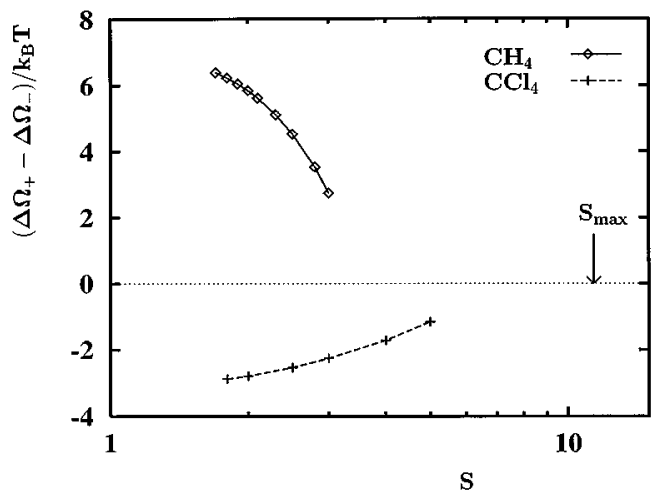

FIG. 11. The sign preference in the reversible work of nucleation of $\mathrm{CH}_{4}$, and $\mathrm{CCl}_{4}$ at $\tilde{T}=0.55$. $r^{\text {ion }}$ is chosen to be the same as the diameter of a $\mathrm{CH}_{4}$ molecule. 


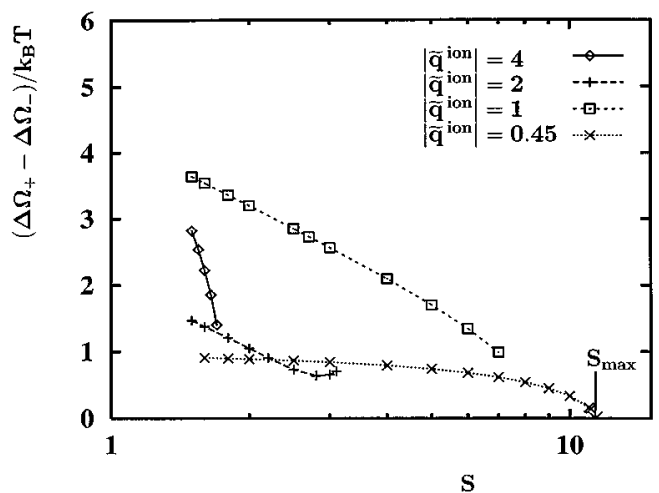

FIG. 12. Effect of $\left|q^{\text {ion }}\right|$ on the sign preference in the reversible work of nucleation of $\mathrm{CS}_{2}$ at $\tilde{T}=0.55$. $\tilde{r}^{\text {ion }}=1$.

$U^{b}(1)$ scales with a dimensionless quantity $b$ :

$$
\tilde{U}(\tilde{1}) \equiv \frac{U^{b}(1)}{k_{B} T_{c}} \sim b,
$$

where

$$
b \equiv \frac{e^{2}}{d k_{B} T_{c}} .
$$

A larger value of $b$ implies a stronger ion-molecule interaction and hence larger reduction in the nucleation barrier. In general, the asymmetry in the ion-molecule interaction is still buried in the dimensionless $\tilde{U}^{b}(\tilde{1}) / b$, the magnitude of $b$ dictating the sensitivity of the system to this asymmetry. In the present case where the polarizabilities and the permanent multipole moments are estimated as above, $\tilde{U}^{b}(\tilde{1})$ for $\mathrm{CCl}_{4}$ differs from that for $\mathrm{CH}_{4}$ only by the value of $b$. Results shown in Fig. 11 are consistent with the values of $b$ given in Table I. Also, Fig. 11 shows that the reversal of the sign of the permanent multipole moments results in reversing the sign preference, as one can see from Eq. (51). It should be noted here that the difference in electronegativity between $\mathrm{Cl}$ and $\mathrm{C}(\sim 0.5)$ is larger than that between $\mathrm{C}$ and $\mathrm{H}(\sim 0.4) .{ }^{24}$ Thus, our prediction on the sign preference for $\mathrm{CCl}_{4}$ is considered to be a lower bound to a true value.

\section{Effect of $r^{\text {ion }}$ and $q^{\text {ion }}$}

In the foregoing, we have shown that the sign preference in the reversible work of nucleation arises from the asymmetric nature in the ion-molecule interaction. One can change the strength of this interaction by changing either $q^{\text {ion }}$ or $r^{\text {ion }}$. Figure 12 shows the effect of $\left|q^{\text {ion }}\right|$ on the sign preference in ion-induced nucleation of $\mathrm{CS}_{2}$, while Fig. 13 shows the effect of $r^{\text {ion }}$. From Figs. 12 and 13, we see that the metastable nucleus reaches its stability limit faster as the electric displacement due to the ion becomes stronger. This follows from the fact that for a given value of the supersaturation $S$, the metastable nucleus becomes larger with increasing ion-molecular interaction, while the critical nucleus shrinks. However, Figs. 12 and 13 show that increasing the ion-molecule interaction does not necessarily increase the sign preference, for when this interaction is increased, so is

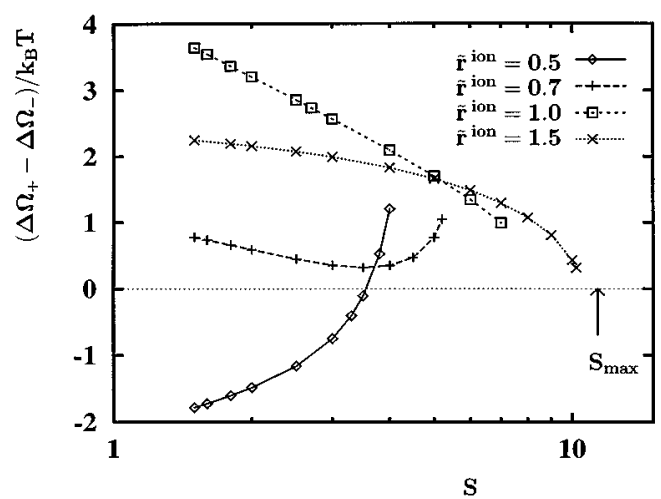

FIG. 13. Effect of $r^{\text {ion }}$ on the sign preference in the reversible work of nucleation of $\mathrm{CS}_{2}$ at $\tilde{T}=0.55$. $\left|\tilde{q}^{\text {ion }}\right|=1$.

the dielectric response of the condensing fluid. In this strong ion-molecule interaction regime, however, contributions from hyperpolarizabilities neglected in Eq. (4) may become significant, ${ }^{25}$ since the interaction energy between the ion and these hyperpolarizabilities scales with some power of $q^{\text {ion }}$ higher than unity. ${ }^{16,17}$ A change in $q^{\text {ion }}$ affects the ionmolecule interaction $U^{b}(1)$ rather uniformly for all the molecules within the whole system, while changing $r^{\text {ion }}$ alters the relative importance of the terms in $U^{b}(1)$ mainly for those molecules close to the ion. This explains the qualitative difference between Figs. 12 and 13.

A rather striking feature to be observed in Fig. 13 is the reversal in the sign preference that occurs at lower supersaturation with $r^{\text {ion }}=0.5 d$. To see its implication, first rewrite the sign preference $\Delta \Omega_{+}-\Delta \Omega_{-}$as follows:

$$
\begin{aligned}
\Delta \Omega_{+}-\Delta \Omega_{-} & =\left(\Omega_{+}^{\text {critical }}-\Omega_{+}^{\text {metastable }}\right)-\left(\Omega_{-}^{\text {critical }}-\Omega_{-}^{\text {metastable }}\right) \\
& =\left(\Omega_{+}^{\text {critical }}-\Omega_{-}^{\text {critical }}\right)-\left(\Omega_{+}^{\text {metastable }}-\Omega_{-}^{\text {metastable }}\right) \\
& =\Delta \Omega^{\text {critical }}-\Delta \Omega^{\text {metastable }} .
\end{aligned}
$$

We show each term in Eq. (55) separately in Fig. 14, which indicates that both $\Delta \Omega^{\text {critical }}$ and $\Delta \Omega^{\text {metastable }}$, in fact, preserve the sign preference that is indicated by $U^{b}(1)$. However,

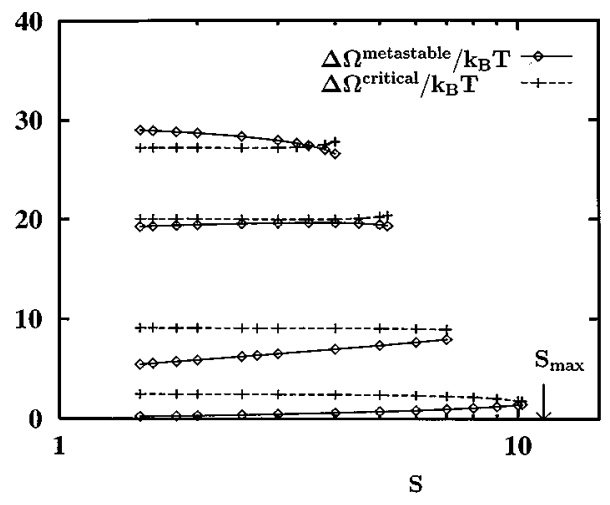

FIG. 14. Variations of $\Delta \Omega^{\text {critical }} / k_{B} T$ and $\Delta \Omega^{\text {metastable }} / k_{B} T$ with supersaturation $S$ for various values of $r^{\text {ion }}$. From the top, they correspond to the case of $\tilde{r}^{\mathrm{inn}}=0.5, \quad \tilde{r}^{\mathrm{ion}}=0.7, \quad \tilde{r}^{\mathrm{ion}}=1.0$, and $\tilde{r}^{\mathrm{ion}}=1.5$, respectively. $\tilde{T}=0.55$ and $\left|\tilde{q}^{\text {ion }}\right|=1$. 
when the ion-molecule interaction is increased near the ion as a result of decreasing $r^{\text {ion }}$, it is counteracted by the dielectric response of the condensing fluid in this region. The effect is more significant for clusters with higher density, namely, for critical nuclei at lower supersaturation, thereby causing $\Delta \Omega^{\text {critical }}$ to become smaller than $\Delta \Omega^{\text {metastable }}$.

In all calculations the sign preference toward a negative ion was recovered as the stability limit is approached. This is only obvious, for

$$
\Delta \Omega_{+}-\Delta \Omega_{-} \sim \Delta \Omega_{+}>0
$$

in this limit as long as the sign preference in the sense mentioned above Eq. (52) is preserved. One can also alter the sign preference in the reversible work by choosing a different value of $q^{\text {ion }}$ or $r^{\text {ion }}$ for a positive ion from that of a negative ion. In this respect, it is important to actually identify the ion involved in experiments on ion-induced nucleation. We note recent progress in this direction reported by Kane et al. ${ }^{26}$

\section{The validity of the model representation}

To evaluate the validity of the current model representation of a molecule, we calculated the dielectric constant $\epsilon_{l}$ of the bulk liquid phase. The intensive state of the liquid is chosen to be the one at vapor-liquid coexistence. When the density profile

$$
n(r) \rightarrow n_{l} \quad \text { as } \quad r \rightarrow \infty
$$

is substituted, Eqs. (18) and (36) yields a solution with a limiting behavior

$$
\epsilon(r) \rightarrow \epsilon_{l} \quad \text { as } \quad r \rightarrow \infty .
$$

We can obtain this $\epsilon_{l}$ by solving Eq. (36) numerically and setting

$$
\epsilon_{l} \approx \epsilon\left(r_{0}\right) .
$$

Alternatively, we may ignore the higher permanent multipole moments and hyperpolarizability to find that

$\epsilon_{l}=1+\frac{16 \pi \mu_{\alpha}^{(P)} \mu_{\alpha}^{(P)}}{9 k_{B} T} n_{l}$

(polar-nonpolarizable molecule),

$\epsilon_{l}=1+\frac{8 \pi \alpha_{\alpha \alpha}}{9} n_{l} \quad$ (nonpolar-polarizable molecule).

Calculated values of $\epsilon_{l}$ are compared in Fig. 15 against the experimental values obtained at $1 \mathrm{~atm} .{ }^{20}$ In the case of $\mathrm{CS}_{2}$, the predicted values of $\epsilon_{l}$ agree well with the data, though the disagreement is quite large $(\sim 130 \%)$ for $\mathrm{CH}_{4}$. In light of the approximations involved in our model representation, the agreement is noteworthy and the model captures many of the most important characteristics of intermolecular or ionmolecule interactions.

\section{SUMMARY AND CONCLUSIONS}

In this paper, we have shown that the sign preference in ion-induced nucleation can be explained in terms of the asymmetric nature of the ion-molecule interaction. Consis-

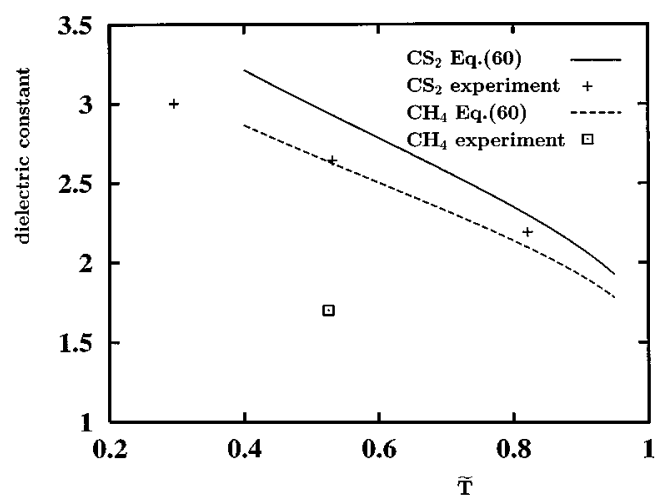

FIG. 15. Comparison of the values of dielectric constant of bulk liquid obtained from the present theory [Eq. (60)] and experiments.

tent treatment of such molecular characteristics is achieved by means of a statistical mechanical density functional theory. Within the framework of a mean field theory, the grand potential is obtained in terms of two order parameters, the particle number density $n(r)$ and the rescaled ion charge $q^{\text {eff }}(r)$, the latter taking account of the dielectric response of the condensing molecules. When the intensive state of the supersaturated vapor is specified, the stationarity condition of the grand potential uniquely determines a critical nucleus and a metastable nucleus for given values of model parameters.

All the molecular parameters used in the present work, if not already available, can be obtained from quantum mechanical calculations. Although the current theory is applicable for both polar and nonpolar materials, we have confined our application of the theory to ion-induced nucleation of $\mathrm{CS}_{2}$ and $\mathrm{CH}_{4}$, for which the required molecular parameters are readily accessible. ${ }^{22,23}$ When the electric displacement due to an ion is sufficiently weak, the calculated reversible work shows a preference consistent with that of the bare ion-molecule interaction potential. In particular, a preference is exhibited toward a negative ion, influencing the nucleation rate by factors of 10 to $10^{2}$ for $\mathrm{CS}_{2}$ and 10 to $10^{5}$ for $\mathrm{CH}_{4}$. The predicted sign preference decreases with increasing supersaturation. Qualitative prediction of the ioninduced nucleation of $\mathrm{CCl}_{4}$ reveals that this substance should exhibit a preference toward positive ions, in agreement with existing data. ${ }^{5}$ Qualitatively different behavior was observed for the predicted sign preference when the electric displacement due to an ion is increased.

Our theory at this stage is at best semiquantitative both in the model representation and in the theoretical treatment. First, we placed polarizabilities and permanent multipole moments at the center of a spherical molecule. Such representation, however, is valid only when the ion-molecule or intermolecular separation is large in comparison to the molecular dimension. To some extent, one could relax this limitation by distributing the polarizabilities and the multipole moments among various sites in a molecule. ${ }^{27-29}$ Part of the molecular symmetry is captured in our model through the tensors representing polarizabilities and permanent multipole moments. However, actual molecular shape is yet another 
important factor in determining the packing structure of molecules around the ion. Proper account of this effect requires an intermolecular potential with an anisotropic repulsive part.

Second, we have characterized an ion by its charge and radius, and the ion-molecule interaction is assumed to be purely electrostatic along with the hard core repulsion at the ion surface. It was shown by Spears ${ }^{25}$ that as long as chemical bonding is negligible, the ion-molecule interaction can indeed be quantitatively treated by an electrostatic model, while the details of the repulsive interaction and the polarizability of the ion were also shown to be important. On the other hand, one would not expect the present theory to be applicable to a system where the chemical nature of the interaction between the ion and molecules plays an important role. ${ }^{30}$ Further complication arises since ions present in the experiments are often complex molecules such as $\mathrm{H}^{+}\left(\mathrm{H}_{2} \mathrm{O}\right)_{n}$ rather than simply ionized atoms. Then, the ion itself must be treated by means of statistical mechanics. Also, we avoided the explicit consideration of the fluctuation of an ion within the nucleus by taking the position of the point charge as the origin. It is expected that at least part of this contribution to the free energy cancels out when taking the difference between two states in obtaining the reversible work. In a more accurate model representation, one would have to treat the system as a binary in which the ion is the second component at extremely low concentration.

Finally, a better treatment of the pair-correlation function than that in a mean field theory, along with the abovementioned model representation of a molecule, will undoubtedly give a better description of the fluid structure within the cluster. Thus the density profile near the ion will exhibit oscillations resembling that near a hard wall, which can either enhance or reduce the oscillatory behavior in the local dielectric constant near the ion. Spontaneous polarization may be observed at the interface as a result of the inhomogeneity in density. Nevertheless, it is clear that some of the most important characteristics of ion-induced nucleation have been captured in the present theory, which forms a basis for explaining this well known phenomenon that has hitherto remained inexplicable within the classical framework.

\section{ACKNOWLEDGMENTS}

This work is supported by National Science Foundation Grant No. ATM-9307603. Part I of this work is Kusaka et al. [J. Chem. Phys. 102, 913 (1995)]. ${ }^{15}$

\section{APPENDIX A: DEFINITIONS OF MULTIPOLE MOMENTS}

We start from the expression for the electrostatic energy $U(1)$ in the external electric field $F_{\alpha}(\mathbf{r})$ of a neutral molecule 1 in a fixed position and orientation: ${ }^{16,17}$

$$
\begin{aligned}
U(1)= & -\mu_{\alpha}^{(P)}(1) F_{\alpha}\left(\mathbf{r}_{1}\right)-\frac{1}{3} \Theta_{\alpha \beta}^{(P)}(1) F_{\alpha \beta}\left(\mathbf{r}_{1}\right)-\frac{1}{15} \Omega_{\alpha \beta \gamma}^{(P)}(1) \\
& \times F_{\alpha \beta \gamma}\left(\mathbf{r}_{1}\right)-\frac{1}{105} \Phi_{\alpha \beta \gamma \delta}^{(P)}(1) F_{\alpha \beta \gamma \delta}\left(\mathbf{r}_{1}\right) \\
& -\frac{1}{2} \alpha_{\alpha \beta}(1) F_{\alpha}\left(\mathbf{r}_{1}\right) F_{\beta}\left(\mathbf{r}_{1}\right) \\
& -\frac{1}{3} A_{\alpha, \beta \gamma}(1) F_{\alpha}\left(\mathbf{r}_{1}\right) F_{\beta \gamma}\left(\mathbf{r}_{1}\right)+\text { h.o., }
\end{aligned}
$$

where $\mu_{\alpha}^{(P)}(1), \Theta_{\alpha \beta}^{(P)}(1), \Omega_{\alpha \beta \gamma}^{(P)}(1)$, and $\Phi_{\alpha \beta \gamma \delta}^{(P)}(1)$ are defined as follows: ${ }^{31}$

$$
\begin{aligned}
& \mu_{\alpha}^{(P)}(1)=\int d \mathbf{r} \rho^{(1)}(\mathbf{r}) x_{\alpha}, \\
& \Theta_{\alpha \beta}^{(P)}(1)=\frac{1}{2} \int d \mathbf{r} \rho^{(1)}(\mathbf{r})\left[3 x_{\alpha} x_{\beta}-r^{2} \delta_{\alpha \beta}\right], \\
& \Omega_{\alpha \beta \gamma}^{(P)}(1)=\frac{1}{2} \int d \mathbf{r} \rho^{(1)}(\mathbf{r})\left[5 x_{\alpha} x_{\beta} x_{\gamma}-r^{2}\left(x_{\alpha} \delta_{\beta \gamma}+x_{\beta} \delta_{\gamma \alpha}\right)\right. \\
&\left.\left.+x_{\gamma} \delta_{\alpha \beta}\right)\right], \\
& \Phi_{\alpha \beta \gamma \delta}^{(P)}(1)= \frac{1}{8} \int d \mathbf{r} \rho^{(1)}(\mathbf{r})\left[35 x_{\alpha} x_{\beta} x_{\gamma} x_{\delta}\right. \\
&-5 r^{2}\left(x_{\alpha} x_{\beta} \delta_{\gamma \delta}+x_{\alpha} x_{\gamma} \delta_{\beta \delta}+x_{\alpha} x_{\delta} \delta_{\beta \gamma}\right. \\
&\left.+x_{\beta} x_{\gamma} \delta_{\alpha \delta}+x_{\beta} x_{\delta} \delta_{\alpha \gamma}+x_{\gamma} x_{\delta} \delta_{\alpha \beta}\right) \\
&\left.+r^{4}\left(\delta_{\alpha \beta} \delta_{\gamma \delta}+\delta_{\alpha \gamma} \delta_{\beta \delta}+\delta_{\alpha \delta} \delta_{\beta \gamma}\right)\right],
\end{aligned}
$$

and, respectively, the permanent electric dipole, quadrupole, octopole, and hexadecapole moments, of the molecule 1 expressed in a laboratory coordinate system $O-x_{1} x_{2} x_{3}$. (See Fig. 1.) Clearly, these quantities are all invariant with respect to any permutation of the indices and become zero when the sum is taken with respect to any two indices. $\rho^{(1)}(\mathbf{r})$ is the charge density distribution in the molecule 1 in the absence of any external field. We introduce the total multipole moments by ${ }^{16,17}$

$$
\begin{aligned}
& \mu_{\alpha}^{(T)}(1) \equiv-\frac{\partial U}{\partial F_{\alpha}}=\mu_{\alpha}^{(P)}(1)+\alpha_{\alpha \beta}(1) F_{\beta}\left(\mathbf{r}_{1}\right) \\
&+\frac{1}{3} A_{\alpha, \beta \gamma}(1) F_{\beta \gamma}\left(\mathbf{r}_{1}\right)+\text { h.o., } \\
& \Theta_{\alpha \beta}^{(T)}(1) \equiv-3 \frac{\partial U}{\partial F_{\alpha \beta}}=\Theta_{\alpha \beta}^{(P)}(1)+A_{\gamma, \alpha \beta}(1) F_{\gamma}\left(\mathbf{r}_{1}\right)+\text { h.o. } \\
& \Omega_{\alpha \beta \gamma}^{(T)} \equiv-15 \frac{\partial U}{\partial F_{\alpha \beta \gamma}}=\Omega_{\alpha \beta \gamma}^{(P)}(1)+\text { h.o., } \\
& \Phi_{\alpha \beta \gamma \delta}^{(T)}(1) \equiv-105 \frac{\partial U}{\partial F_{\alpha \beta \gamma \delta}}=\Phi_{\alpha \beta \gamma \delta}^{(P)}(1)+\text { h.o. }
\end{aligned}
$$

Since $A_{\gamma, \alpha \beta}$ is symmetric with respect to $\alpha$ and $\beta$ and $A_{\gamma, \alpha \alpha} \equiv 0,{ }^{16,17}$ it is clear that the total multipole moments possess the same properties as those of the permanent multiple moments mentioned above. In fact, we can define the total multipole moments through similar relations to those given in Eq. (A2) with the superscript $(P)$ replaced by $(T)$. Finally, we can rewrite Eq. (A1) to obtain Eq. (4) by means of Eq. (A3).

\section{APPENDIX B: $U^{\mathrm{mp}}(\mathbf{1 , 2})$}

We start from Eq. (9). $F_{\alpha}^{(2)}\left(\mathbf{r}_{1}\right)$ is given in terms of the electrostatic potential $\Psi^{(2)}(\mathbf{r})$ created by the total multipole moments on the molecule 2 :

$$
F_{\alpha}^{(2)}\left(\mathbf{r}_{1}\right)=-\left.\frac{\partial \Psi^{(2)}(\mathbf{r})}{\partial x_{\alpha}}\right|_{\mathbf{r}=\mathbf{r}_{1}} .
$$


Denoting the $\rho^{(2)}(\mathbf{r})$ the charge density distribution in the molecule 2 in the presence of the ion and the other molecules,

$$
\Psi^{(2)}(\mathbf{r})=\int d \mathbf{r}^{\prime} \frac{\rho^{(2)}\left(\mathbf{r}^{\prime}\right)}{\left|\mathbf{r}-\mathbf{r}^{\prime}\right|} .
$$

We expand the denominator around $\mathbf{r}^{\prime}=\mathbf{r}_{2}$ and use Eq. (A2) with the superscript $(P)$ replaced by $(T)$. Taking the spatial derivatives of the resulting expression with respect to $\mathbf{r}$ and setting $\mathbf{r}=\mathbf{r}_{1}$, as indicated by Eq. (6), we obtain for a neutral molecule

$$
\begin{aligned}
F_{\alpha}^{(2)}\left(\mathbf{r}_{1}\right)= & T_{\alpha \beta} \mu_{\beta}^{(T)}(2)+\frac{1}{3} T_{\alpha \beta \gamma} \Theta_{\beta \gamma}^{(T)}(2)+\frac{1}{15} T_{\alpha \beta \gamma \delta} \Omega_{\beta \gamma \delta}^{(T)}(2) \\
& +\frac{1}{105} T_{\alpha \beta \gamma \delta \epsilon} \Phi_{\beta \gamma \delta \epsilon}^{(T)}(2)+O\left(r_{12}^{-7}\right), \\
F_{\alpha \beta}^{(2)}\left(\mathbf{r}_{1}\right)= & -T_{\alpha \beta \gamma} \mu_{\gamma}^{(T)}(2)-\frac{1}{3} T_{\alpha \beta \gamma \delta} \Theta_{\gamma \delta}^{(T)}(2)-\frac{1}{15} T_{\alpha \beta \gamma \delta \epsilon} \Omega_{\gamma \delta \epsilon}^{(T)} \\
& \times(2)+O\left(r_{12}^{-7}\right), \\
F_{\alpha \beta \gamma}^{(2)}\left(\mathbf{r}_{1}\right)= & T_{\alpha \beta \gamma \delta} \mu_{\delta}^{(T)}(2)+\frac{1}{3} T_{\alpha \beta \gamma \delta \epsilon} \Theta_{\delta \epsilon}^{(T)}(2)+O\left(r_{12}^{-7}\right), \\
F_{\alpha \beta \gamma \delta}^{(2)}\left(\mathbf{r}_{1}\right)= & -T_{\alpha \beta \gamma \delta \epsilon} \mu_{\epsilon}^{(T)}(2)+O\left(r_{12}^{-7}\right),
\end{aligned}
$$

where

$$
\begin{aligned}
& \left.T_{\alpha \beta} \equiv \frac{\partial^{2} r^{-1}}{\partial x_{\alpha} \partial x_{\beta}}\right|_{\mathbf{r}=\mathbf{r}_{12}}, \\
& \left.T_{\alpha \beta \gamma} \equiv \frac{\partial^{3} r^{-1}}{\partial x_{\alpha} \partial x_{\beta} \partial x_{\gamma}}\right|_{\mathbf{r}=\mathbf{r}_{12}}, \\
& \left.T_{\alpha \beta \gamma \delta} \equiv \frac{\partial^{4} r^{-1}}{\partial x_{\alpha} \partial x_{\beta} \partial x_{\gamma} \partial x_{\delta}}\right|_{\mathbf{r}=\mathbf{r}_{12}}, \\
& \left.T_{\alpha \beta \gamma \delta \epsilon} \equiv \frac{\partial^{5} r^{-1}}{\partial x_{\alpha} \partial x_{\beta} \partial x_{\gamma} \partial x_{\delta} \partial x_{\epsilon}}\right|_{\mathbf{r}=\mathbf{r}_{12}} .
\end{aligned}
$$

Using Eq. (B3) in Eq. (9), we obtain

$U^{\mathrm{mp}}(1,2)$

$$
\begin{aligned}
= & -T_{\alpha \beta} \mu_{\alpha}^{(T)}(1) \mu_{\beta}^{(T)}(2)-\frac{1}{3} T_{\alpha \beta \gamma}\left\{\mu_{\alpha}^{(T)}(1) \Theta_{\beta \gamma}^{(T)}(2)\right. \\
& \left.-\Theta_{\alpha \beta}^{(T)}(1) \mu_{\gamma}^{(T)}(2)\right\}-T_{\alpha \beta \gamma \delta}\left\{\frac{1}{15} \mu_{\alpha}^{(T)}(1) \Omega_{\beta \gamma \delta}^{(T)}(2)\right. \\
& \left.-\frac{1}{9} \Theta_{\alpha \beta}^{(T)}(1) \Theta_{\gamma \delta}^{(T)}(2)+\frac{1}{15} \Omega_{\alpha \beta \gamma}^{(T)}(1) \mu_{\delta}^{(T)}(2)\right\}-T_{\alpha \beta \gamma \delta \epsilon} \\
& \times\left\{\frac{1}{105} \mu_{\alpha}^{(T)}(1) \Phi_{\beta \gamma \delta \epsilon}^{(T)}(2)-\frac{1}{45} \Theta_{\alpha \beta}^{(T)}(1) \Omega_{\gamma \delta \epsilon}^{(T)}(2)\right. \\
& \left.+\frac{1}{45} \Omega_{\alpha \beta \gamma}^{(T)}(1) \Theta_{\delta \epsilon}^{(T)}(2)-\frac{1}{105} \Phi_{\alpha \beta \gamma \delta}^{(T)}(1) \mu_{\epsilon}^{(T)}(2)\right\} .
\end{aligned}
$$

When $\mathbf{r}_{1}$ and $\mathbf{r}_{2}$ are exchanged, $T_{\alpha \beta \gamma}$ and $T_{\alpha \beta \gamma \delta \epsilon}$ change their signs while $T_{\alpha \beta}$ and $T_{\alpha \beta \gamma \delta}$ remain unaffected, since $\mathbf{r}_{12}=-\mathbf{r}_{21}$. Thus, $U^{\mathrm{mp}}(1,2)$ is symmetric in 1 and 2 , a property used in arriving at Eqs. (35) and (36) from Eq. (32).

\section{APPENDIX C: THE ION-MOLECULE INTERACTION ENERGY}

We choose the coordinate system in which the position vector $\mathbf{r}_{1}$ of the molecule 1 is parallel to the $x_{3}$ axis. (See Fig.
1.) The only nonzero components of the electric field and its spatial gradients at $\mathbf{r}_{1}$, resulting from a point charge $q$ at the origin, are

$$
\begin{aligned}
& F_{3}=\frac{q}{r_{1}^{2}}, \\
& F_{11}=F_{22}=\frac{q}{r_{1}^{3}}, \\
& F_{33}=-\frac{2 q}{r_{1}^{3}}, \\
& F_{113}=F_{223}=-\frac{3 q}{r_{1}^{4}}, \\
& F_{333}=\frac{6 q}{r_{1}^{4}}, \\
& F_{1111}=F_{2222}=-\frac{9 q}{r_{1}^{5}}, \\
& F_{3333}=-\frac{24 q}{r_{1}^{5}}, \\
& F_{1133}=F_{2233}=\frac{12 q}{r_{1}^{5}}, \\
& F_{1}^{5},
\end{aligned}
$$

and those obtained by any permutation of their indices. Using the symmetry and traceless properties discussed in Appendix $\mathrm{A}$ of the multipole moments and the polarizabilities, we therefore obtain

$$
\begin{aligned}
& \mu_{\alpha}(1) F_{\alpha}\left(\mathbf{r}_{1}\right)=\frac{q}{r_{1}^{2}} \mu_{3}(1), \\
& \Theta_{\alpha \beta}(1) F_{\alpha \beta}\left(\mathbf{r}_{1}\right)=-\frac{3 q}{r_{1}^{3}} \Theta_{33}(1), \\
& \Omega_{\alpha \beta \gamma}(1) F_{\alpha \beta \gamma}\left(\mathbf{r}_{1}\right)=\frac{15 q}{r_{1}^{4}} \Omega_{333}(1), \\
& \Phi_{\alpha \beta \gamma \delta}(1) F_{\alpha \beta \gamma \delta}\left(\mathbf{r}_{1}\right)=-\frac{105 q}{r_{1}^{5}} \Phi_{3333}(1), \\
& \alpha_{\alpha \beta}(1) F_{\alpha}\left(\mathbf{r}_{1}\right) F_{\beta}\left(\mathbf{r}_{1}\right)=\frac{q^{2}}{r_{1}^{4}} \alpha_{33}(1), \\
& A_{\alpha, \beta \gamma}(1) F_{\alpha}\left(\mathbf{r}_{1}\right) F_{\beta \gamma}\left(\mathbf{r}_{1}\right)=-\frac{3 q^{2}}{r_{1}^{5}} A_{3,33}(1) .
\end{aligned}
$$

The first four equations are valid for both total and permanent multipole moments. Using Eq. (C2) and setting $q=q^{\text {ion }}$, we rewrite Eq. (8) to obtain Eq. (14). Similar procedures are followed with $q=q^{\text {eff }}\left(r_{1}\right)$ to obtain Eq. (19) from Eq. (4) and Eq. (20) from Eq. (5). 
Finally, using Eq. (C1) in Eq. (A3) with $q=q^{\text {eff }}\left(r_{1}\right)$, we obtain

$$
\begin{aligned}
\mu_{3}^{(T)}(1)= & \mu_{3}^{(P)}(1)+q^{\mathrm{eff}}\left(r_{1}\right)\left(\frac{1}{r_{1}^{2}} \alpha_{33}(1)-\frac{1}{r_{1}^{3}} A_{3,33}(1)\right) \\
& + \text { h.o., } \\
\Theta_{33}^{(T)}(1)= & \Theta_{33}^{(P)}(1)+\frac{q^{\text {eff }}\left(r_{1}\right)}{r_{1}^{2}} A_{3,33}(1)+\text { h.o., }
\end{aligned}
$$

$$
\begin{aligned}
& \Omega_{333}^{(T)}(1)=\Omega_{333}^{(P)}(1)+\text { h.o., } \\
& \Phi_{3333}^{(T)}(1)=\Phi_{3333}^{(P)}(1)+\text { h.o., }
\end{aligned}
$$

which is substituted into Eq. (14) to obtain Eq. (21).

\section{APPENDIX D: DERIVATION OF EQ. (36)}

We start from Eq. (32), which is rewritten here as

$$
\begin{aligned}
\Omega\left[n, q^{\mathrm{eff}}\right]= & \int d \mathbf{r}_{1} f^{d}\left(n\left(r_{1}\right)\right)-\int d \mathbf{r}_{1} n\left(r_{1}\right)\left\{\mu+k_{B} T \log \left[Z_{R}\left(r_{1}\right)\right]\right\}+\int d 1 n\left(r_{1}\right) m(1)\left[q^{\mathrm{ion}}-q^{\mathrm{eff}}\left(r_{1}\right)\right] u^{T}(1) \\
& +\frac{1}{2} \iint d \mathbf{r}_{1} d \mathbf{r}_{2} n\left(r_{1}\right) n\left(r_{2}\right) H\left(r_{12}-d\right) \phi^{\mathrm{att}}\left(r_{12}\right)+\frac{1}{2} \iint d 1 d 2 n\left(r_{1}\right) n\left(r_{2}\right) m(1) m(2) H\left(r_{12}-d\right) \phi^{\mathrm{mp}}(1,2) .
\end{aligned}
$$

Consider an infinitesimal change in $q^{\text {eff }}\left(r_{1}\right)$ while $n\left(r_{1}\right)$ is fixed. Noting that $\phi^{\mathrm{mp}}(1,2)$ is symmetric in 1 and 2 ,

$$
\begin{aligned}
\delta \Omega= & -k_{B} T \int d \mathbf{r}_{1} n\left(r_{1}\right) \frac{1}{Z_{R}\left(r_{1}\right)} \frac{\partial Z_{R}\left(r_{1}\right)}{\partial q^{\mathrm{eff}}\left(r_{1}\right)} \delta q^{\mathrm{eff}}\left(r_{1}\right)-\int d 1 n\left(r_{1}\right) m(1) u^{T}(1) \delta q^{\mathrm{eff}}\left(r_{1}\right)+\int d 1 n\left(r_{1}\right) \\
& \times\left[q^{\mathrm{ion}}-q^{\mathrm{eff}}\left(r_{1}\right)\right] u^{T}(1) \delta m(1)+\int d 1 n\left(r_{1}\right) m(1)\left[q^{\mathrm{ion}}-q^{\mathrm{eff}}\left(r_{1}\right)\right] \frac{\partial u^{T}(1)}{\partial q^{\mathrm{eff}}\left(r_{1}\right)} \delta q^{\mathrm{eff}}\left(r_{1}\right)+\iint d 1 d 2 n\left(r_{1}\right) n\left(r_{2}\right) m(2) \\
& \times H\left(r_{12}-d\right) \phi^{\mathrm{mp}}(1,2) \delta m(1)+\iint d 1 d 2 n\left(r_{1}\right) n\left(r_{2}\right) m(1) m(2) H\left(r_{12}-d\right) \frac{\partial \phi^{\mathrm{mp}}(1,2)}{\partial q^{\mathrm{eff}}\left(r_{1}\right)} \delta q^{\mathrm{eff}}\left(r_{1}\right) .
\end{aligned}
$$

From Eqs. (19)-(21), (28), and (29)

$$
\begin{aligned}
& \frac{\partial u^{T}(1)}{\partial q^{\mathrm{eff}}\left(r_{1}\right)}=-u^{\mathrm{pol}}(1), \\
& \frac{1}{Z_{R}\left(r_{1}\right)} \frac{\partial Z_{R}\left(r_{1}\right)}{\partial q^{\mathrm{eff}}\left(r_{1}\right)}=-\frac{1}{k_{B} T}\left\langle u^{T}(1)\right\rangle_{\hat{R}_{1}}, \\
& \delta m(1)=\frac{1}{k_{B} T} m(1)\left[\left\langle u^{T}(1)\right\rangle_{\hat{R}_{1}}-u^{T}(1)\right] \delta q^{\mathrm{eff}}\left(r_{1}\right) .
\end{aligned}
$$

Thus Eq. (D1) finally yields

$$
\begin{aligned}
\frac{\delta \Omega}{\delta q^{\mathrm{eff}}=}= & \frac{n\left(r_{1}\right)}{k_{B} T}\left[[ q ^ { \mathrm { eff } } ( r _ { 1 } ) - q ^ { \mathrm { ion } } ] \left\{\left[\left\langle u^{T}(1)^{2}\right\rangle_{R_{1}}-\left\langle u^{T}(1)\right\rangle_{\hat{R}_{1}}^{2}\right]\right.\right. \\
& \left.+k_{B} T\left\langle u^{\mathrm{pol}}(1)\right\rangle_{\hat{R}_{1}}\right\}-\int d \mathbf{r}_{2} n\left(r_{2}\right) H\left(r_{12}-d\right) \\
& \times\left\{\left[\left\langle\phi^{\mathrm{mp}}(1,2) u^{T}(1)\right\rangle_{\hat{R}_{1} \hat{R}_{2}}-\left\langle\phi^{\mathrm{mp}}(1,2)\right\rangle_{\hat{R}_{1} \hat{R}_{2}}\right.\right. \\
& \left.\left.\left.\times\left\langle u^{T}(1)\right\rangle_{\hat{R}_{1}}\right]-k_{B} T\left\langle\frac{\partial \phi^{\mathrm{mp}}(1,2)}{\partial q^{\mathrm{eff}}\left(r_{1}\right)}\right\rangle_{\hat{R}_{1} \hat{R}_{2}}\right\}\right] . \quad \text { (D4) }
\end{aligned}
$$

Assuming that $n\left(r_{1}\right)$ is nonzero everywhere in the system, we finally arrive at Eq. (36).

\section{APPENDIX E: ANALYTICAL INTEGRATIONS OF EQS. (32), (35), AND (36)}

When Eq. (28) is employed, it becomes convenient to introduce the local coordinate system $O^{(j)}-x_{1}^{(j)} x_{2}^{(j)} x_{3}^{(j)}$ at the center of the molecule $j$, in which the $x_{3}^{(j)}$ axis is parallel to $\mathbf{r}_{j}$. The orientation of the molecule $j$ is determined by specifying the Euler angle $\left(\phi_{j}, \theta_{j}, \psi_{j}\right)$ of the body fixed coordinate system $O^{(B j)}-x_{1}^{(B j)} x_{2}^{(B j)} x_{3}^{(B j)}$ on the molecule $j$ with respect to the local coordinate system. We have chosen $O-x_{1} x_{2} x_{3}$ so that the $x_{3}$ axis is parallel to the $x_{3}^{(1)}$ axis. (See Fig. 1.)

Since any tensor in the form of $G_{333 \cdots 3}(1)$ is invariant under a rotation around the $x_{3}^{(1)}$ axis specified by $\phi_{1}$, it is readily seen from Eqs. (19)-(21) that $U(1), u^{\mathrm{pol}}(1)$, and $u^{T}(1)$ are all independent of $\phi_{1}$. For clarity, we introduce new notations:

$$
\begin{aligned}
& U_{0}\left(\xi_{1}\right)=U(1), \\
& u_{0}^{\mathrm{pol}}\left(\xi_{1}\right)=u^{\mathrm{pol}}(1), \\
& u_{0}^{T}\left(\xi_{1}\right)=u^{T}(1) .
\end{aligned}
$$


We use the notation $\xi_{j}$ to denote the position $\mathbf{r}_{j}$ and the orientation $\left(\theta_{j}, \psi_{j}\right)$ of the molecule $j$. The orientation alone is denoted by $\omega_{j}$, which reduces to $\theta_{j}$ in the case of a linear molecule. Performing the integration with respect to $\phi_{1}$ in $Z_{R}\left(r_{1}\right)$, we define $m_{0}\left(\xi_{1}\right)$ through

$$
m(1)=\frac{1}{2 \pi Z_{0}\left(r_{1}\right)} \exp \left\{-\frac{U_{0}\left(\xi_{1}\right)}{k_{B} T}\right\} \equiv \frac{1}{2 \pi} m_{0}\left(\xi_{1}\right),
$$

where

$$
Z_{0}\left(r_{1}\right) \equiv \int d \omega_{1} \exp \left\{-\frac{U_{0}\left(\xi_{1}\right)}{k_{B} T}\right\}=\frac{1}{2 \pi} Z_{R}\left(r_{1}\right) .
$$

Using Eqs. (E1)-(E3) in Eqs. (32), (35), and (36) we obtain

$$
\begin{aligned}
\Omega\left[n, q^{\mathrm{eff}}\right]= & \int d \mathbf{r}_{1} f^{d}\left(n\left(r_{1}\right)\right)-\int d \mathbf{r}_{1} n\left(r_{1}\right)\left\{\mu+k_{B} T \log \left[2 \pi Z_{0}\left(r_{1}\right)\right]\right\}+\int d \mathbf{r}_{1} n\left(r_{1}\right)\left[q^{\mathrm{ion}}-q^{\mathrm{eff}}\left(r_{1}\right)\right]\left\langle u_{0}^{T}\left(\xi_{1}\right)\right\rangle_{\omega_{1}} \\
& +\frac{1}{2} \iint d \mathbf{r}_{1} d \mathbf{r}_{2} n\left(r_{1}\right) n\left(r_{2}\right) H\left(r_{12}-d\right) \phi^{\mathrm{att}}\left(r_{12}\right)+\frac{1}{2} \iint d \mathbf{r}_{1} d \mathbf{r}_{2} n\left(r_{1}\right) n\left(r_{2}\right) H\left(r_{12}-d\right)\left\langle\phi_{0}^{\mathrm{mp}}\left(\xi_{1}, \xi_{2}\right)\right\rangle_{\omega_{1} \omega_{2}},
\end{aligned}
$$

$$
\begin{aligned}
0= & \mu^{d}\left(n\left(r_{1}\right)\right)-\left\{\mu+k_{B} T \log \left[2 \pi Z_{0}\left(r_{1}\right)\right]\right\} \\
& +\left[q^{\text {ion }}-q^{\text {eff }}\left(r_{1}\right)\right]\left\langle u_{0}^{T}\left(\xi_{1}\right)\right\rangle_{\omega_{1}} \\
& +\int d \mathbf{r}_{2} n\left(r_{2}\right) H\left(r_{12}-d\right) \phi^{\text {att }}\left(r_{12}\right) \\
& +\int d \mathbf{r}_{2} n\left(r_{2}\right) H\left(r_{12}-d\right)\left\langle\phi_{0}^{\mathrm{mp}}\left(\xi_{1}, \xi_{2}\right)\right\rangle_{\omega_{1} \omega_{2}},
\end{aligned}
$$

and

$$
\begin{aligned}
0= & {\left[q^{\mathrm{eff}}\left(r_{1}\right)-q^{\mathrm{ion}}\right]\left\{\left[\left\langle u_{0}^{T}\left(\xi_{1}\right)^{2}\right\rangle_{\omega_{1}}-\left\langle u_{0}^{T}\left(\xi_{1}\right)\right\rangle_{\omega_{1}}^{2}\right]\right.} \\
& \left.+k_{B} T\left\langle u_{0}^{\mathrm{pol}}\left(\xi_{1}\right)\right\rangle_{\omega_{1}}\right\}-\int d \mathbf{r}_{2} n\left(r_{2}\right) H\left(r_{12}-d\right) \\
& \times\left\{\left[\left\langle\phi_{0}^{\mathrm{mp}}\left(\xi_{1}, \xi_{2}\right) u_{0}^{T}\left(\xi_{1}\right)\right\rangle_{\omega_{1} \omega_{2}}\right.\right.
\end{aligned}
$$

$$
\begin{aligned}
& \left.-\left\langle\phi_{0}^{\mathrm{mp}}\left(\xi_{1}, \xi_{2}\right)\right\rangle_{\omega_{1} \omega_{2}}\left\langle u_{0}^{T}\left(\xi_{1}\right)\right\rangle_{\omega_{1}}\right] \\
& \left.-k_{B} T\left\langle\frac{\partial \phi_{0}^{\mathrm{mp}}\left(\xi_{1}, \xi_{2}\right)}{\partial q^{\mathrm{eff}}\left(r_{1}\right)}\right\rangle_{\omega_{1} \omega_{2}}\right\},
\end{aligned}
$$

respectively. For arbitrary functions $G_{a}\left(\xi_{1}\right)$ and $G_{b}\left(\xi_{1}, \xi_{2}\right)$, we have defined their angular average by

$$
\left\langle G_{a}\left(\xi_{1}\right)\right\rangle_{\omega_{1}} \equiv \int d \omega_{1} m\left(\xi_{1}\right) G_{a}\left(\xi_{1}\right)
$$

and

$\left\langle G_{b}\left(\xi_{1}, \xi_{2}\right)\right\rangle_{\omega_{1} \omega_{2}} \equiv \iint d \omega_{1} d \omega_{2} m\left(\xi_{1}\right) m\left(\xi_{2}\right) G_{b}\left(\xi_{1}, \xi_{2}\right)$,

respectively. $\phi_{0}^{\mathrm{mp}}\left(\xi_{1}, \xi_{2}\right)$ is defined by

$$
\begin{aligned}
\phi_{0}^{\mathrm{mp}}\left(\xi_{1}, \xi_{2}\right) \equiv & \frac{1}{(2 \pi)^{2}} \int_{0}^{2 \pi} d \phi_{1} \int_{0}^{2 \pi} d \phi_{2} \phi^{\mathrm{mp}}(1,2)=-T_{\alpha \beta} \mu_{\alpha}^{(T)}\left(\xi_{1}\right) \mu_{\beta}^{(T)}\left(\xi_{2}\right)-\frac{1}{3} T_{\alpha \beta \gamma}\left\{\mu_{\alpha}^{(T)}\left(\xi_{1}\right) \theta_{\beta \gamma}^{(T)}\left(\xi_{2}\right)-\Theta_{\alpha \beta}^{(T)}\left(\xi_{1}\right) \mu_{\gamma}^{(T)}\left(\xi_{2}\right)\right\} \\
& -T_{\alpha \beta \gamma \delta}\left\{\frac{1}{15} \mu_{\alpha}^{(T)}\left(\xi_{1}\right) \Omega_{\beta \gamma \delta}^{(T)}\left(\xi_{2}\right)-\frac{1}{9} \Theta_{\alpha \beta}^{(T)}\left(\xi_{1}\right) \Theta_{\gamma \delta}^{(T)}\left(\xi_{2}\right)+\frac{1}{15} \Omega_{\alpha \beta \gamma}^{(T)}\left(\xi_{1}\right) \mu_{\delta}^{(T)}\left(\xi_{2}\right)\right\}-T_{\alpha \beta \gamma \delta \epsilon}\left\{\frac{1}{105} \mu_{\alpha}^{(T)}\left(\xi_{1}\right)\right. \\
& \left.\times \Phi_{\beta \gamma \delta \epsilon}^{(T)}\left(\xi_{2}\right)-\frac{1}{45} \Theta_{\alpha \beta}^{(T)}\left(\xi_{1}\right) \Omega_{\gamma \delta \epsilon}^{(T)}\left(\xi_{2}\right)+\frac{1}{45} \Omega_{\alpha \beta \gamma}^{(T)}\left(\xi_{1}\right) \Theta_{\delta \epsilon}^{(T)}\left(\xi_{2}\right)-\frac{1}{105} \Phi_{\alpha \beta \gamma \delta}^{(T)}\left(\xi_{1}\right) \mu_{\epsilon}^{(T)}\left(\xi_{2}\right)\right\}
\end{aligned}
$$

where the second equality follows from Eq. (B5) and the definitions

$$
\begin{aligned}
& \mu_{\alpha}^{(T)}\left(\xi_{j}\right) \equiv \frac{1}{2 \pi} \int_{0}^{2 \pi} d \phi_{j} \mu_{\alpha}^{(T)}(j), \quad \Theta_{\alpha \beta}^{(T)}\left(\xi_{j}\right) \equiv \frac{1}{2 \pi} \int_{0}^{2 \pi} d \phi_{j} \Theta_{\alpha \beta}^{(T)}(j), \\
& \Omega_{\alpha \beta \gamma}^{(T)}\left(\xi_{j}\right) \equiv \frac{1}{2 \pi} \int_{0}^{2 \pi} d \phi_{j} \Omega_{\alpha \beta \gamma}^{(T)}(j), \quad \Phi_{\alpha \beta \gamma \delta}^{(T)}\left(\xi_{j}\right) \equiv \frac{1}{2 \pi} \int_{0}^{2 \pi} d \phi_{j} \Phi_{\alpha \beta \gamma \delta}^{(T)}(j) .
\end{aligned}
$$

We next rewrite $u_{0}^{T}\left(\xi_{1}\right)$ and $\phi_{0}^{\mathrm{mp}}\left(\xi_{1}, \xi_{2}\right)$ in terms of the tensor components in the local coordinate system. When referred to the local coordinate system $O^{(j)}-x_{1}^{(j)} x_{2}^{(j)} x_{3}^{(j)}$, these tensors defined by Eq. (E10) are axially symmetric around the $x_{3}^{(j)}$ axis. Hence the only nonzero components are ${ }^{17}$

$$
\begin{aligned}
& \mu_{3}^{(T j)}\left(\xi_{j}\right), \quad \Theta_{11}^{(T j)}\left(\xi_{j}\right)=\Theta_{22}^{(T j)}\left(\xi_{j}\right)=-\frac{1}{2} \Theta_{33}^{(T j)}\left(\xi_{j}\right), \quad \Omega_{113}^{(T j)}\left(\xi_{j}\right)=\Omega_{223}^{(T j)}\left(\xi_{j}\right)=-\frac{1}{2} \Omega_{333}^{(T j)}\left(\xi_{j}\right), \\
& \Phi_{1111}^{(T j)}\left(\xi_{j}\right)=\Phi_{2222}^{(T j)}\left(\xi_{j}\right)=\frac{3}{8} \Phi_{3333}^{(T j)}\left(\xi_{j}\right), \quad \Phi_{1122}^{(T j)}\left(\xi_{j}\right)=\frac{1}{8} \Phi_{3333}^{(T j)}\left(\xi_{j}\right), \quad \Phi_{1133}^{(T j)}\left(\xi_{j}\right)=\Phi_{2233}^{(T j)}\left(\xi_{j}\right)=-\frac{1}{2} \Phi_{3333}^{(T j)}\left(\xi_{j}\right),
\end{aligned}
$$


and those obtained by any permutation of the indices. We add the superscript $j$ for the tensor components expressed in the local coordinate system $O^{(j)}-x_{1}^{(j)} x_{2}^{(j)} x_{3}^{(j)}$. For the particular choice of the coordinate system shown in Fig. $1, O-x_{1} x_{2} x_{3}$ is related to $O^{(1)}-x_{1}^{(1)} x_{2}^{(1)} x_{3}^{(1)}$ by a linear translation. Thus, the tensor components expressed in the former are the same as those expressed in the latter. In particular,

$$
\mu_{3}^{(T)}\left(\xi_{1}\right)=\mu_{3}^{(T 1)}\left(\xi_{1}\right), \quad \Theta_{33}^{(T)}\left(\xi_{1}\right)=\Theta_{33}^{(T 1)}\left(\xi_{1}\right), \quad \Omega_{333}^{(T)}\left(\xi_{1}\right)=\Omega_{333}^{(T 1)}\left(\xi_{1}\right), \quad \Phi_{3333}^{(T)}\left(\xi_{1}\right)=\Phi_{3333}^{(T 1)}\left(\xi_{1}\right) .
$$

Other components are readily obtained from Eq. (E11). From Eqs. (14), (E1), (E10), and (E12)

$$
u_{0}^{T}\left(\xi_{1}\right)=-\frac{1}{r_{1}^{2}} \mu_{3}^{(T 1)}\left(\xi_{1}\right)+\frac{1}{r_{1}^{3}} \Theta_{33}^{(T 1)}\left(\xi_{1}\right)-\frac{1}{r_{1}^{4}} \Omega_{333}^{(T 1)}\left(\xi_{1}\right)+\frac{1}{r_{1}^{5}} \Phi_{3333}^{(T 1)}\left(\xi_{1}\right)
$$

For the molecule 2,

$$
\begin{aligned}
& \mu_{\alpha}^{(T)}\left(\xi_{2}\right)=a_{\beta \alpha} \mu_{\beta}^{(T 2)}\left(\xi_{2}\right), \quad \Theta_{\alpha \beta}^{(T)}\left(\xi_{2}\right)=a_{\gamma \alpha} a_{\delta \beta} \Theta_{\gamma \delta}^{(T 2)}\left(\xi_{2}\right), \quad \Omega_{\alpha \beta \gamma}^{(T)}\left(\xi_{2}\right)=a_{\delta \alpha} a_{\epsilon \beta} a_{\mu \gamma} \Omega_{\delta \epsilon \mu}^{(T 2)}\left(\xi_{2}\right), \\
& \Phi_{\alpha \beta \gamma \delta}^{(T)}\left(\xi_{2}\right)=a_{\epsilon \alpha} a_{\mu \beta} a_{\tau \gamma} a_{\rho \delta} \Phi_{\epsilon \mu \tau \rho}^{(T 2)}\left(\xi_{2}\right),
\end{aligned}
$$

where $a_{\alpha \beta}$ is the orthogonal transformation matrix from $O-x_{1} x_{2} x_{3}$ to $O^{(2)}-x_{1}^{(2)} x_{2}^{(2)} x_{3}^{(2)}$. Due to the axial symmetry of the system around the $x_{3}$ axis, we may set

$$
a=\left(\begin{array}{ccc}
\cos \theta & 0 & -\sin \theta \\
0 & 1 & 0 \\
\sin \theta & 0 & \cos \theta
\end{array}\right),
$$

where $\theta$ is the angle between the $x_{3}$ axis and the $x_{3}^{(2)}$ axis. Using Eqs. (E11), (E12), (E14), and (E15), we may integrate Eq. (E9) with respect to $\cos \theta$ to obtain

$$
\begin{aligned}
\Phi_{0}^{\mathrm{mp}}\left(\xi_{1}, \xi_{2}\right)= & -\mu_{3}^{(T 1)}\left(\xi_{1}\right) \mu_{3}^{(T 2)}\left(\xi_{2}\right) \frac{\partial}{\partial(\cos \theta)} A_{\mu-\mu}\left(r_{1}, r_{2}, \cos \theta\right)-\frac{3}{2} \Theta_{33}^{(T 1)}\left(\xi_{1}\right) \mu_{3}^{(T 2)}\left(\xi_{2}\right) \frac{\partial}{\partial(\cos \theta)} A_{\Theta-\mu}\left(r_{1}, r_{2}, \cos \theta\right) \\
& -\frac{3}{2} \mu_{3}^{(T 1)}\left(\xi_{1}\right) \Theta_{33}^{(T 2)}\left(\xi_{2}\right) \frac{\partial}{\partial(\cos \theta)} A_{\Theta-\mu}\left(r_{2}, r_{1}, \cos \theta\right)+\frac{1}{2} \Omega_{333}^{(T 1)}\left(\xi_{1}\right) \mu_{3}^{(T 2)}\left(\xi_{2}\right) \frac{\partial}{\partial(\cos \theta)} A_{\Omega-\mu}\left(r_{1}, r_{2}, \cos \theta\right) \\
& +\frac{3}{4} \Theta_{33}^{(T 1)}\left(\xi_{1}\right) \Theta_{33}^{(T 2)}\left(\xi_{2}\right) \frac{\partial}{\partial(\cos \theta)} A_{\Theta-\Theta}\left(r_{1}, r_{2}, \cos \theta\right)+\frac{1}{2} \mu_{3}^{(T 1)}\left(\xi_{1}\right) \Omega_{333}^{(T 2)}\left(\xi_{2}\right) \frac{\partial}{\partial(\cos \theta)} A_{\Omega-\mu}\left(r_{2}, r_{1}, \cos \theta\right) \\
& +\frac{5}{8} \Phi_{3333}^{(T 1)}\left(\xi_{1}\right) \mu_{3}^{(T 2)}\left(\xi_{2}\right) \frac{\partial}{\partial(\cos \theta)} A_{\Phi-\mu}\left(r_{1}, r_{2}, \cos \theta\right)-\frac{5}{4} \Omega_{333}^{(T 1)}\left(\xi_{1}\right) \Theta_{33}^{(T 2)}\left(\xi_{2}\right) \\
& \times \frac{\partial}{\partial(\cos \theta)} A_{\Omega-\Theta}\left(r_{1}, r_{2}, \cos \theta\right)-\frac{5}{4} \Theta_{33}^{(T 1)}\left(\xi_{1}\right) \Omega_{333}^{(T 2)}\left(\xi_{2}\right) \frac{\partial}{\partial(\cos \theta)} A_{\Omega-\Theta}\left(r_{2}, r_{1}, \cos \theta\right)+\frac{5}{8} \mu_{3}^{(T 1)}\left(\xi_{1}\right) \\
& \times \Phi_{3333}^{(T 2)}\left(\xi_{2}\right) \frac{\partial}{\partial(\cos \theta)} A_{\Phi-\mu}\left(r_{2}, r_{1}, \cos \theta\right),
\end{aligned}
$$

where

$$
\begin{aligned}
& A_{\mu-\mu}\left(r_{1}, r_{2}, \cos \theta\right)=\frac{1}{r_{12}^{3}}\left(\cos ^{2} \theta-1\right), \\
& A_{\Theta-\mu}\left(r_{1}, r_{2}, \cos \theta\right)=\frac{1}{r_{12}^{5}}\left(r_{2} \cos \theta-r_{1}\right)\left(\cos ^{2} \theta-1\right),
\end{aligned}
$$$$
A_{\Omega-\mu}\left(r_{1}, r_{2}, \cos \theta\right)
$$$$
=\left[\frac{1}{r_{12}^{5}}-\frac{5}{r_{12}^{7}}\left(r_{2} \cos \theta-r_{1}\right)^{2}\right]\left(\cos ^{2} \theta-1\right),
$$$$
A_{\Phi-\mu}\left(r_{1}, r_{2}, \cos \theta\right)=\left[\frac{3}{r_{12}^{7}}-\frac{7}{r_{12}^{9}}\left(r_{2} \cos \theta-r_{1}\right)^{2}\right]
$$$$
\times\left(r_{2} \cos \theta-r_{1}\right)\left(\cos ^{2} \theta-1\right),
$$

$$
\begin{aligned}
A_{\Theta-\Theta}\left(r_{1}, r_{2}, \cos \theta\right) \\
\quad=\left[\frac{4}{r_{12}^{5}} \cos \theta+\frac{5}{r_{12}^{7}} r_{1} r_{2}\left(\cos ^{2} \theta-1\right)\right]\left(\cos ^{2} \theta-1\right),
\end{aligned}
$$

$$
\begin{aligned}
A_{\Omega-\Theta}\left(r_{1}, r_{2}, \cos \theta\right)= & \frac{1}{r_{12}^{7}}\left(r_{2}-r_{1} \cos \theta\right)+\frac{2}{r_{12}^{7}} \\
& \times\left(r_{2} \cos \theta-r_{1}\right) \cos \theta-\frac{7}{r_{12}^{9}} \\
& \left.\times\left(r_{2} \cos \theta-r_{1}\right)^{2}\left(r_{2}-r_{1} \cos \theta\right)\right] \\
& \times\left(\cos ^{2} \theta-1\right) .
\end{aligned}
$$


Recall that Eq. (C3) is obtained for the coordinate system in which the $x_{3}$ axis is parallel to $\mathbf{r}_{1}$. Since the $x_{3}^{(j)}$ axis in the local coordinate system of the molecule $j$ is parallel to $\mathbf{r}_{j}$, we have similar relations:

$$
\begin{aligned}
\mu_{3}^{(T j)}\left(\xi_{j}\right)= & \mu_{3}^{(P j)}\left(\xi_{j}\right)+q^{\mathrm{eff}}\left(r_{j}\right) \\
& \times\left(\frac{1}{r_{j}^{2}} \alpha_{33}^{(j)}\left(\xi_{j}\right)-\frac{1}{r_{j}^{3}} A_{3,33}^{(j)}\left(\xi_{j}\right)\right)+\text { h.o., } \\
\Theta_{33}^{(T j)}\left(\xi_{j}\right)= & \Theta_{33}^{(P j)}\left(\xi_{j}\right)+\frac{q^{\mathrm{eff}}\left(r_{j}\right)}{r_{j}^{2}} A_{3,33}^{(j)}\left(\xi_{j}\right)+\text { h.o., } \\
\Omega_{333}^{(T j)}\left(\xi_{j}\right)= & \Omega_{333}^{(P j)}\left(\xi_{j}\right)+\text { h.o., } \\
\Phi_{3333}^{(T j)}\left(\xi_{j}\right)= & \Phi_{3333}^{(P j)}\left(\xi_{j}\right)+\text { h.o., }
\end{aligned}
$$

where the superscript $j$ has the same significance as before. The functional form of each tensor given by Eq. (E18) is identical for every molecule, as a result of the spherical symmetry of the system. Note that the tensor components on the RHS of Eq. (E18) are expressed in the local coordinate system $O^{(j)}-x_{1}^{(j)} x_{2}^{(j)} x_{3}^{(j)}$ and can be expressed in terms of those in the body fixed coordinate system $O^{(B j)}-x_{1}^{(B j)} x_{2}^{(B j)} x_{3}^{(B j)}$ and the Euler angle $\left(\phi_{j}=0, \theta_{j}, \psi_{j}\right)$ of the latter with respect to the former. Although a molecule is supposed to be a sphere in our model representation, part of the molecular symmetry is captured in the model through the symmetry of these tensors.

For arbitrary function $G_{c}\left(r_{1}, r_{2}, \cos \theta\right)$, we have

$$
\begin{aligned}
\int & d \mathbf{r}_{2} H\left(r_{12}-d\right) G_{c}\left(r_{1}, r_{2}, \cos \theta\right) \\
= & 2 \pi \int_{r^{\mathrm{ion}}+(d / 2)}^{\infty} r_{2}^{2} d r_{2} \int_{-1}^{c\left(r_{1}, r_{2}\right)} d(\cos \theta) G_{c}\left(r_{1}, r_{2}, \cos \theta\right)
\end{aligned}
$$

and

$$
\begin{aligned}
\int & \int d \mathbf{r}_{1} d \mathbf{r}_{2} H\left(r_{12}-d\right) G_{c}\left(r_{1}, r_{2}, \cos \theta\right) \\
= & 8 \pi^{2} \int_{r^{\mathrm{ion}_{+}}+(d / 2)}^{r_{0}} r_{1}^{2} d r_{1} \int_{r^{\mathrm{ion}_{+}(d / 2)}}^{\infty} r_{2}^{2} d r_{2} \\
& \times \int_{-1}^{c\left(r_{1}, r_{2}\right)} d(\cos \theta) G_{c}\left(r_{1}, r_{2}, \cos \theta\right),
\end{aligned}
$$

where $r_{0}$ is the radius of the system boundary. $c\left(r_{1}, r_{2}\right)$ is unity except when the molecule 2 stays inside the spherical shell $r_{L}<r<r 1+d$, where $r_{L}$ is the larger of $r^{\text {ion }}+d / 2$ and $r_{1}-d$. In this case,

$$
c\left(r_{1}, r_{2}\right)=\frac{r_{1}^{2}+r_{2}^{2}-d^{2}}{2 r_{1} r_{2}},
$$

which is the cosine of the angle between $\mathbf{r}_{1}$ and $\mathbf{r}_{2}$ when the molecule 2 is in contact with the molecule 1 . This is the consequence of the hard sphere exclusion represented by $H\left(r_{12}-d\right)$. When Eq. (E21) is used along with Eq. (E16) and the relation

$$
\phi^{\mathrm{att}}\left(r_{12}\right)=\frac{\partial}{\partial(\cos \theta)}\left[-\frac{\epsilon^{\mathrm{att}} d^{6}}{4 r_{1} r_{2}\left(r_{1}^{2}+r_{2}^{2}-2 r_{1} r_{2} \cos \theta\right)^{2}}\right],
$$

which follows from Eq. (3), integrals with respect to $\cos \theta$ in Eqs. (E4)-(E6) become analytically tractable, reducing the dimensionality of the integrals in Eq. (E4) to at most four: two over $\omega_{1}$ in calculating the angular average of the total multipole moments on the molecule 1 , and the other two over $r_{1}$ and $r_{2}$. It is not necessary to calculate the angular average of the total multipole moments on the molecule 2, for they are identical as functions of the ion-molecule distance to those for the molecule 1. Similarly, integrations in Eqs. (E5) and (E6) are at most three dimensional.

Finally, the quantities defined in Eqs. (E17) become zero at $\cos \theta= \pm 1$. Thus, the integration of $\phi_{0}^{\mathrm{mp}}\left(\xi_{1}, \xi_{2}\right)$ with respect to $\cos \theta$ vanishes unless $r_{2}$ is in the spherical shell described above. Hence the contribution to the free energy density or the local dielectric constant at $r_{1}$ through $\phi^{\mathrm{mp}}(1,2)$ comes from only those molecules within this spherical shell. Also, $c\left(r_{1}, r_{2}\right)$ approaches unity as $r_{1} \rightarrow \infty$; hence this contribution tends to cancel at this limit, the fact used in the discussion of Fig. 8 in Sec. IV A. These are the results of our mean field approximation.

${ }^{1}$ C. T. R. Wilson, Philos. Trans. R. Soc. London Ser. A 189, 265 (1897).

${ }^{2}$ L. B. Loeb, A. F. Kip, and A. W. Einarsson, J. Chem. Phys. 6, 264 (1938).

${ }^{3}$ L. Scharrer, Ann. Phys. 35, 619 (1939).

${ }^{4}$ D. R. White and J. L. Kassner, Jr., J. Aerosol Sci. 2, 201 (1971).

${ }^{5}$ H. Rabeony and P. Mirabel, J. Phys. Chem. 91, 1815 (1987).

${ }^{6}$ M. Adachi, K. Okuyama, and J. H. Seinfeld, J. Aerosol Sci. 23, 327 (1992).

${ }^{7}$ F. He and P. K. Hopke, J. Chem. Phys. 99, 9972 (1993).

${ }^{8}$ H. Rabeony and P. Mirabel, J. Chim. Phys. 83, 219 (1986).

${ }^{9}$ J. L. Katz, J. A. Fisk, and V. M. Chakarov, J. Chem. Phys. 101, 2309 (1994).

${ }^{10}$ L. Y. Chan and V. A. Mohnen, J. Atmos. Sci. 37, 2323 (1980).

${ }^{11}$ S. H. Suck, J. Chem. Phys. 75, 5090 (1981).

${ }^{12}$ A. I. Rusanov, and F. M. Kuni, J. Colloid Interface Sci. 100, 264 (1984).

${ }^{13}$ A. K. Shchěkin, A. I. Rusanov, and F. M. Kuni, Kolloidn. Zh. 46, 535 (1984).

${ }^{14}$ J. J. Thomson, Conduction of Electricity Through Gases (Cambridge University, Cambridge, 1906).

${ }^{15}$ I. Kusaka, Z.-G. Wang, and J. H. Seinfeld, J. Chem. Phys. 102, 913 (1995).

${ }^{16}$ A. D. Buckingham, Adv. Chem. Phys. 12, 107 (1967).

${ }^{17}$ A. D. McLean and M. Yoshimine, J. Chem. Phys. 47, 1927 (1967).

${ }^{18}$ R. Evans, Adv. Phys. 28, 143 (1979).

${ }^{19}$ N. F. Carnahan and K. E. Starling, J. Chem. Phys. 51, 635 (1969); 53, 600 (1970).

${ }^{20}$ CRC Handbook of Chemistry and Physics, 74th ed., edited by D. R. Lide (CRC, Boca Raton, FL, 1993).

${ }^{21}$ C. Huiszoon, Mol. Phys. 58, 865 (1986).

${ }^{22}$ G. Maroulis, Chem. Phys. Lett. 199, 250 (1992).

${ }^{23}$ G. Maroulis, Chem. Phys. Lett. 226, 420 (1994).

${ }^{24}$ L. Pauling, General Chemistry (Dover, New York, 1988).

${ }^{25}$ K. G. Spears, J. Chem. Phys. 57, 1850 (1972).

${ }^{26}$ D. Kane, G. M. Daly, and M. S. El-Shall, J. Phys. Chem. 99, 7867 (1995).

${ }^{27}$ A. J. Stone, Chem. Phys. Lett. 83, 233 (1981).

${ }^{28}$ A. J. Stone and M. Alderton, Mol. Phys. 56, 1047 (1985).

${ }^{29}$ A. J. Stone, Mol. Phys. 56, 1065 (1985).

${ }^{30}$ A. W. Castleman, Jr., Adv. Colloid Interface Sci. 10, 73 (1979).

${ }^{31}$ D. E. Stogryn and A. P. Stogryn, Mol. Phys. 11, 371 (1966). 